\title{
CONSEQUENCES OF THE LAKSHMIBAI-SANDHYA THEOREM: THE UBIQUITY OF PERMUTATION PATTERNS IN SCHUBERT CALCULUS AND RELATED GEOMETRY
}

\author{
HIRAKU ABE AND SARA BILLEY
}

\begin{abstract}
In 1990, Lakshmibai and Sandhya published a characterization of singular Schubert varieties in flag manifolds using the notion of pattern avoidance. This was the first time pattern avoidance was used to characterize geometrical properties of Schubert varieties. Their results are very closely related to work of Haiman, Ryan and Wolper, but Lakshmibai-Sandhya were the first to use that language exactly. Pattern avoidance in permutations was used historically by Knuth, Pratt, Tarjan, and others in the 1960's and 1970's to characterize sorting algorithms in computer science. Lascoux and Schützenberger also used pattern avoidance to characterize vexillary permutations in the 1980's. Now, there are many geometrical properties of Schubert varieties that use pattern avoidance as a method for characterization including Gorenstein, factorial, local complete intersections, and properties of Kazhdan-Lusztig polynomials. These are what we call consequences of the Lakshmibai-Sandhya theorem. We survey the many beautiful results, generalizations, and remaining open problems in this area. We highlight the advantages of using pattern avoidance characterizations in terms of linear time algorithms and the ease of access to the literature via Tenner's Database of Permutation Pattern Avoidance. This survey is based on lectures by the second author at Osaka, Japan 2012 for the Summer School of the Mathematical Society of Japan based on the topic of Schubert calculus.
\end{abstract}

\section{INTRODUCTION}

Modern Schubert calculus is the study of effective methods to compute the expansion coefficients for the cup product of cohomology classes of Schubert varieties:

$$
\left[X_{u}\right] \cdot\left[X_{v}\right]=\sum c_{u, v}^{w}\left[X_{w}\right]
$$

These coefficients $c_{u, v}^{w}$ are called structure constants with respect to the Schubert classes $\left[X_{w}\right]$, and it is known that the structure constants are non-negative integers. In fact, each $c_{u, v}^{w}$ is the intersection number of three Schubert varieties $X_{u}, X_{v}$ and $X_{w_{0}}$; they count the number of points of the intersection of those three varieties placed in generic positions. Observe that this is both a combinatorial and a geometrical statement.

For Schubert varieties in Grassmannians, we already have many tools for computing the structure constants for the cup product: Littlewood-Richardson tableaux, Yamanouchi words, Knutson-Tao puzzles, Vakil's toric degenerations. In general, we have not found analogs of all these beautiful tools for other types of Schubert varieties. We need to understand both the combinatorics and geometry of Schubert varieties in order to do Schubert calculus for all types of Schubert varieties.

In this article, we will focus on the combinatorics and geometry related to the tangent spaces of Schubert varieties and characterizations of smoothness and rational smoothness. The mathematical tools we will use also arise in Schubert calculus, but we will not make the connections explicit. For the record, the most explicit connection between characterizations of

Date: March 27, 2018.

The second author was partially supported by grant DMS-1101017 from the NSF. 
smoothness and Schubert calculus come from Kumar's criterion and the Kostant polynomials. See [8, 63, 93, for more details.

We begin with a review of Schubert varieties in flag manifolds. Then we will present the celebrated Lakshmibai-Sandhya Theorem characterizing smooth Schubert varieties using permutation pattern avoidance. We will give a total of 10 properties of Schubert varieties in flag manifolds that are completely characterized by pattern avoidance or a variation on that theme. We describe a method for extending permutation pattern avoidance to all Coxeter groups and discuss some geometrical properties characterized by Coxeter pattern avoidance more generally. We give pointers to some useful computational tools for studying Schubert geometry and beyond. Finally, we present many open problems in this area.

We want to highlight the fact that there are computational advantages of using permutation patterns to characterize interesting properties such as smoothness of Schubert varieties. Naively, avoiding a finite set of patterns of length at most $k$ leads to a polynomial time algorithm of $O\left(n^{k}\right)$ by brute force testing of all $k$-subsets. As $k$ and $n$ get large, such algorithm is intractable. In fact, deciding if one permutation is contained in another is an NP-complete problem [18. Remarkably, Guillemot and Marx [51] recently showed that for every permutation $v \in S_{k}$ there exists an algorithm to test if $w \in S_{n}$ contains $v$ which runs in linear time, $O(n)$ ! This is a major improvement over brute force verification. It is often far from obvious that an $O(n)$ time algorithm exists for the geometric or algebraic properties characterized by pattern avoidance in this paper.

Another major advantage of permutation pattern characterizations is that they provide efficient fingerprints for theorems [15]. Tenner's Database of Permutation Pattern Avoidance (DPPA) provides a growing collection of known properties characterized by patterns with references to the literature 92. This allows researchers to connect new theorems and conjectures with known results in a format free of language or notational differences.

\section{Preliminaries}

\subsection{The Flag Manifold.}

Definition 2.1. A complete flag $F_{\bullet}=\left(F_{1}, \ldots, F_{n}\right)$ in $\mathbb{C}^{n}$ is a nested sequence of vector spaces such that $\operatorname{dim}\left(F_{i}\right)=i$ for $1 \leq i \leq n$. A flag $F_{\bullet}$ is determined by an ordered basis $\left\langle f_{1}, f_{2}, \ldots, f_{n}\right\rangle$ where $F_{i}=\operatorname{span}\left\langle f_{1}, \ldots, f_{i}\right\rangle$.

Let $e_{1}, e_{2}, \ldots, e_{n}$ be the standard basis for $\mathbb{C}^{n}$. The base flag is $E_{\bullet}=\left(E_{1}, E_{2}, \ldots, E_{n}\right)$ where $E_{i}=\left\langle e_{1}, e_{2}, \ldots, e_{i}\right\rangle$. Let $F_{\bullet}$ be any flag given by the ordered basis $\left\langle f_{1}, f_{2}, \ldots, f_{n}\right\rangle$. Writing each basis element $f_{i}$ as a column vector in terms of the $e_{i}$ 's, we obtain an $n \times n$-nonsingular matrix whose column vectors are the basis $f_{1}, \cdots, f_{n}$. In this presentation, we can multiply the matrix by a non-zero scalar or we can add the $i$-th column to the $j$-th column where $i<j$ and it still represents the same flag. So, a flag can always be presented by a matrix in canonical form; the lowest non-zero entry of each column is 1 , and the entries to its right are all zeros.

Example 2.2. The following two matrices represent the same flag $F_{\bullet}=\left\langle 2 e_{1}+e_{2}, 2 e_{1}+\right.$ $\left.e_{3}, \quad 7 e_{1}+e_{4}, \quad e_{1}\right\rangle$ :

$$
\left[\begin{array}{llll}
6 & 4 & 9 & 0 \\
3 & 0 & 0 & 1 \\
0 & 2 & 1 & 0 \\
0 & 0 & 1 & 0
\end{array}\right] \sim\left[\begin{array}{llll}
2 & 2 & 7 & 1 \\
1 & 0 & 0 & 0 \\
0 & 1 & 0 & 0 \\
0 & 0 & 1 & 0
\end{array}\right]
$$

The right hand side is the canonical form. 
It also follows that two non-singular matrices represent the same flag if and only if one is the other multiplied by an upper triangular matrix. That is, we have an identification $\mathcal{F} l_{n}(\mathbb{C})=G L_{n}(\mathbb{C}) / \tilde{B}$ where $\tilde{B} \subset G L_{n}(\mathbb{C})$ is the set of invertible upper triangular matrices. Similarly, we can rescale any invertible matrix by the inverse of its determinant and get another matrix representing the same flag. Hence, letting $B$ be the set of upper triangular matrices in $S L_{n}(\mathbb{C})$, we see that

$$
\mathcal{F} l_{n}(\mathbb{C})=G L_{n}(\mathbb{C}) / \tilde{B}=S L_{n}(\mathbb{C}) / B .
$$

2.2. Flags and Permutations. If a flag is written in canonical form, the leading 1's form a permutation matrix. This matrix is called the position of the flag $F_{\bullet}$ with respect to the base flag $E_{\bullet}$, and is denoted by $\operatorname{position}\left(E_{\bullet}, F_{\bullet}\right)$.

Example 2.3.

$$
F_{\bullet}=\left\langle 2 e_{1}+e_{2}, \quad 2 e_{1}+e_{3}, \quad 7 e_{1}+e_{4}, \quad e_{1}\right\rangle \approx\left[\begin{array}{cccc}
2 & 2 & 7 & 1 \\
1 & 0 & 0 & 0 \\
0 & 1 & 0 & 0 \\
0 & 0 & 1 & 0
\end{array}\right]
$$

Note that there are many ways to represent a permutation; as a bijection from $[n]:=$ $\{1,2, \ldots, n\}$ to itself, matrix notation, two-line notation, one-line notation, rank table, diagram, string diagram, reduced word etc. Each of these representations is useful in some way or another for the study of Schubert varieties so we advise the reader to become comfortable with all of them simultaneously and choose the right one for the proof at hand. Note, we have not found much use for cycle notation for permutations in this context so we will not ever use that notation here.

To be precise, we use the following notation: for a permutation $w:[n] \rightarrow[n]$ in the symmetric group $S_{n}$, we denote by the same symbol $w=w_{1} w_{2} \ldots w_{n}$ the permutation matrix which has 1's in the $\left(w_{j}, j\right)$-th entries for $1 \leq j \leq n$ and 0's elsewhere. Permutation multiplication is consistent with matrix multiplication using this notation. In particular, if $t_{i j}$ is the transposition interchanging $i$ and $j$, then the one-line notation for $w t_{i j}$ agrees with $w$ in all positions except $i$ and $j$ where the entries are switched. The permutation $t_{i j} w$ has the values $i$ and $j$ switched.

The rank table $r k(w)$ is obtained from the matrix $w$ by setting

$$
r k(w)[i, j]=\#\{h \in[j]: w(h) \in[i]\},
$$

i.e. the rank of the submatrix of $w$ with lower right corner $[i, j]$ and upper left corner $[1,1]$.

A string diagram of a permutation for $w$ is a braid with the strings proceeding from the initial ordering to the permuted order given by $w=w_{1} w_{2} \ldots w_{n}$ in such a way that no three strings cross at any point. A wiring diagram is a string diagram with exactly one crossing on each row. A wiring diagram in which no two strings cross twice is said to be reduced. Starting at the top of a reduced wiring diagram, one can read off the index of the first string in each crossing to obtain a corresponding reduced word. All reduced words for $w$ have the same length, denoted $\ell(w)$. Furthermore, the length of $w$ is the number of inversions for $w$, $\ell(w)=\#\{w(i)>w(j): i<j\}$.

The diagram of a permutation $w$ is obtained from the matrix of $w^{-1}$ by removing all cells in an $n \times n$ array which are weakly to the right or below a 1 in $w^{-1}$. The remaining cells form the diagram $D(w)$. The cells of $D(w)$ are in bijection with the inversions of $w$. One can recover $w$ either from its diagram or its inversion set. It is unfortunate that the diagram is defined in terms of $w^{-1}$, but that is the most common convention in the literature [70. 


\section{Example 2.4.}

$$
\begin{aligned}
& {\left[\begin{array}{llll}
0 & 0 & 0 & 1 \\
1 & 0 & 0 & 0 \\
0 & 1 & 0 & 0 \\
0 & 0 & 1 & 0
\end{array}\right]=\left[\begin{array}{llll}
1 & 2 & 3 & 4 \\
2 & 3 & 4 & 1
\end{array}\right]=2341=\left[\begin{array}{llll}
0 & 0 & 0 & 1 \\
1 & 1 & 1 & 2 \\
1 & 2 & 2 & 3 \\
1 & 2 & 3 & 4
\end{array}\right]} \\
& \text { matrix two-line one-line rank } \\
& \text { notation notation notation table } \\
& * \cdot \cdot 1234 \\
& \text { *. } \cdot \cdot \cdot \quad=\text { YH }=(1,2,3) \\
& \text {. . . } 2341 \\
& \begin{array}{cc}
\text { diagram of a } & \text { string diagram } \\
\text { permutation } & \text { woduced } \\
\text { word }
\end{array}
\end{aligned}
$$

2.3. Schubert Cells and Schubert Varieties in $\mathcal{F} l_{n}(\mathbb{C})$. For a permutation $w \in S_{n}$, the Schubert cell $C_{w}\left(E_{\bullet}\right) \subset \mathcal{F} l_{n}(\mathbb{C})$ is the set of all flags $F_{\bullet}$ with $\operatorname{position}\left(E_{\bullet}, F_{\bullet}\right)=w$. Equivalently, we can write $C_{w}\left(E_{\bullet}\right)$ as

$$
C_{w}\left(E_{\bullet}\right)=\left\{F_{\bullet} \in \mathcal{F} l_{n}(\mathbb{C}) \mid \operatorname{dim}\left(E_{i} \cap F_{j}\right)=\operatorname{rk}(w)[i, j] \text { for all } 1 \leq i, j \leq n\right\} .
$$

Note, the flag $w_{\bullet}$ represented by the permutation matrix for $w$ is in $C_{w}$ by the rank conditions.

\section{Example 2.5.}

$$
F_{\bullet}=\left[\begin{array}{cccc}
2 & 2 & 7 & 1 \\
1 & 0 & 0 & 0 \\
0 & 1 & 0 & 0 \\
0 & 0 & 1 & 0
\end{array}\right] \in C_{2341}=\left\{\left[\begin{array}{cccc}
x & y & z & 1 \\
1 & \cdot & \cdot & \cdot \\
\cdot & 1 & \cdot & \cdot \\
\cdot & \cdot & 1 & \cdot
\end{array}\right]: x, y, z \in \mathbb{C}\right\}
$$

It is easy to observe the following properties for each permutation $w$.

(i) The dimension of a Schubert cell is $\operatorname{dim}_{\mathbb{C}}\left(C_{w}\right)=\ell(w)$.

(ii) The indeterminates for the canonical matrices in $C_{w}$ all lie in the entries of the diagram $D\left(w^{-1}\right)$.

(iii) $C_{w}=\tilde{B} \cdot w_{\bullet}$ is a $\tilde{B}$-orbit using the left $\tilde{B}$ action on flags given by multiplication of matrices. See Example 2.6.

Example 2.6. For arbitrary $b_{i, j}$ 's with $b_{1,1}, b_{2,2}, b_{3,3}, b_{4,4}$ non-zero, we have

$$
\left[\begin{array}{cccc}
b_{1,1} & b_{1,2} & b_{1,3} & b_{1,4} \\
0 & b_{2,2} & b_{2,3} & b_{2,4} \\
0 & 0 & b_{3,3} & b_{3,4} \\
0 & 0 & 0 & b_{4,4}
\end{array}\right]\left[\begin{array}{cccc}
0 & 0 & 0 & 1 \\
1 & 0 & 0 & 0 \\
0 & 1 & 0 & 0 \\
0 & 0 & 1 & 0
\end{array}\right]=\left[\begin{array}{cccc}
b_{1,2} & b_{1,3} & b_{1,4} & b_{1,1} \\
b_{2,2} & b_{2,3} & b_{2,4} & 0 \\
0 & b_{3,3} & b_{3,4} & 0 \\
0 & 0 & b_{4,4} & 0
\end{array}\right] \in C_{2341} .
$$

Definition 2.7. The Schubert variety $X_{w}\left(E_{\bullet}\right)$ of a permutation $w$ is defined to be the closure of $C_{w}\left(E_{\bullet}\right)$ under the Zariski topology. As in the case for Schubert cells, $X_{w}\left(E_{\bullet}\right)$ can be written by the rank conditions:

$$
X_{w}\left(E_{\bullet}\right)=\left\{F_{\bullet} \in \mathcal{F} l_{n} \mid \operatorname{dim}\left(E_{i} \cap F_{j}\right) \geq \operatorname{rk}(w)[i, j] \text { for all } 1 \leq i, j \leq n\right\} .
$$

Example 2.8.

$$
\left.\left[\begin{array}{cccc}
1 & 0 & 0 & 0 \\
0 & * & * & 1 \\
0 & 1 & 0 & 0 \\
0 & 0 & 1 & 0
\end{array}\right] \in X_{2341}\left(E_{\bullet}\right)=\left\{\begin{array}{cccc}
* & * & * & 1 \\
1 & 0 & 0 & 0 \\
0 & 1 & 0 & 0 \\
0 & 0 & 1 & 0
\end{array}\right]\right\}
$$


2.4. Combinatorics and Geometry. Since Schubert cells are $\tilde{B}$-orbits, Schubert varieties are $\tilde{B}$-invariant by their definition. So each Schubert variety is equal to a disjoint union of Schubert cells

$$
X_{w}=\bigcup_{v \leq w} C_{v} .
$$

Thus, the containment relation on Schubert varieties $X_{v} \subset X_{w}$ defines a partial order on permutations $v \leq w$. This partial order has a nice description: for a permutation $w$ and integers $1 \leq i<j \leq n$, we say $w<w t_{i j}$ if $w(i)<w(j)$. Bruhat order (discovered by Ehresmann 1934 [39], see also Chevalley 1958 [29]) is defined to be the transitive closure of this relation.

Example 2.9. The following is the Hasse diagram of the Bruhat order on permutations in $S_{3}$.

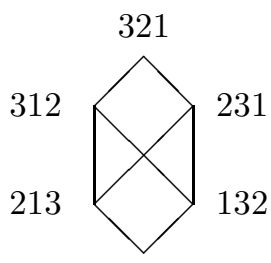

123

The Hasse diagram of $S_{n}$ is self dual, rank symmetric and rank unimodal.

Example 2.10. The Hasse diagram of $S_{4}$ is drawn in Figure 1

One of the benefits of Bruhat order is a description of the Poincaré polynomials of Schubert varieties. More precisely, the Poincaré polynomial for $H^{*}\left(X_{w}\right)$ is given by

$$
P_{w}(t)=\sum_{v \leq w} t^{2 l(v)}
$$

Because only even exponents appear in the Poincaré polynomials above, we often abuse notation and define

$$
P_{w}(t)=\sum_{v \leq w} t^{l(v)} .
$$

Example 2.11. For $w=3412$, the following permutations are in the interval below 3412 in Bruhat order.

$$
\begin{array}{lc}
4: & (3412) \\
3: & (3142)(3214)(1432)(2413) \\
2: & (3124)(1342)(2143)(2314)(1423) \\
1: & (2134)(1243)(1324) \\
0: & (1234)
\end{array}
$$

So $P_{3412}(t)=1+3 t+5 t^{2}+4 t^{3}+t^{4}$. One can see that the Schubert variety $X_{3412}$ is not smooth since its Poincaré polynomial is not symmetric (palindromic) which implies that Poincaré duality does not hold for $H^{*}\left(X_{3412}\right)$.

There are several interesting things about Bruhat order. We will encounter some of them in the rest of the paper. We will focus on the relationship between singularities of Schubert varieties and pattern avoidance of permutations. 


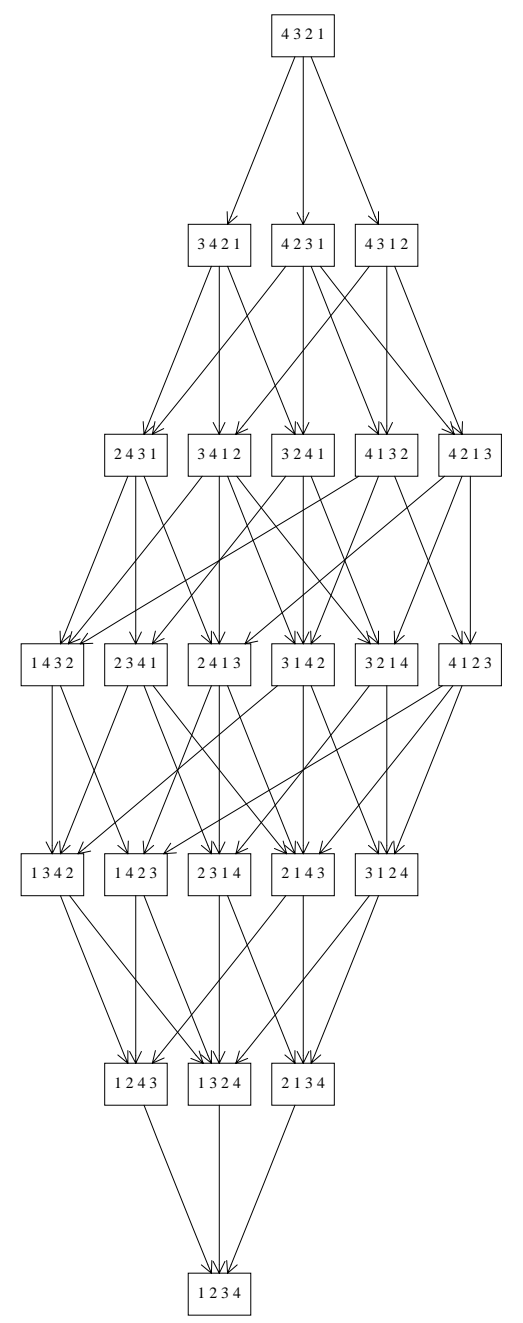

Figure 1. The Hasse diagram of $S_{4}$

We leave to the reader the following exercises.

(1) The boundary of $X_{w}$ has irreducible components given by the Schubert varieties $X_{v}$ such that $v<w$ such that $\ell(v)=\ell(w)-1$.

(2) $C_{w}$ is a dense open set in $X_{w}$.

(3) $X_{w}$ embeds into a product of projective spaces via Plücker coordinates. A matrix is mapped under this embedding to the list all of its lower left minors in a given order.

(4) If $w_{0}=[n, n-1, \ldots, 1]$, then $G L_{n} / \tilde{B}=X_{w_{0}}$.

(5) The point $w_{0}$ has an affine neighborhood $C_{w_{0}}$ of dimension $\left(\begin{array}{l}n \\ 2\end{array}\right)$ and a local coordinate system. A generic point $g$ has an affine neighborhood $g w_{0} C_{w_{0}}$ in $\mathcal{F} l_{n}$.

(6) $G L_{n}$ acts transitively on the points in the flag manifold so it is a manifold and a projective variety.

(7) The flag manifold is smooth (i.e. non-singular at every point). 


\section{Smooth Schubert varieties}

Say we wish to determine which Schubert varieties are smooth and which are not. There are several combinatorial and geometrical observations which makes this determination easier to characterize than a typical variety.

First, an affine variety is smooth at a point if the dimension of its tangent space equals the dimension of the variety near that point. If the variety is given in terms of the vanishing of certain polynomials, then one can check the dimension of the tangent space by computing the rank of the Jacobian matrix for those polynomials evaluated at the point. The rank is smaller than expected if and only if all minors of a certain size vanish. Thus, the set of points where the variety is not smooth is itself a variety called the singular locus.

A priori, to determine if a variety is smooth at every point, one must check the dimension of the tangent space at every point. For Schubert varieties, we make an easy observation. A point $p \in C_{v} \subset X_{w}$ is singular in $X_{w}$ if and only if every point in $C_{v}$ is singular in $X_{w}$ since the Schubert cell $C_{v}$ is a $\tilde{B}$-orbit. Recalling that the singular locus of a variety is a closed set, the equality (10) implies that each Schubert variety $X_{w}$ is smooth if and only if $X_{w}$ is smooth at the identity matrix $I$. One can check the singularity at the identity by writing down the defining equations of $X_{w}$ around an affine neighborhood of $X_{w}$ around $I$ (for example, $\left.X_{w} \cap w_{0} C_{w_{0}}\right)$ and check the rank of the Jacobian matrix of the defining polynomials. However, there is another way which provides a more unified tool for the study of the singularity of Schubert varieties using Lie algebras.

3.1. Lie algebras and tangent spaces of Schubert varieties. Recall from Section 2.1 that the flag variety can be identified with the quotient of a semisimple algebraic group:

$$
\mathcal{F} l_{n}=G L_{n}(\mathbb{C}) / \tilde{B}=S L_{n}(\mathbb{C}) / B
$$

where $\tilde{B}$ is the set of upper triangular matrices in $G L_{n}(\mathbb{C})$ and $B=\tilde{B} \cap S L_{n}(\mathbb{C})$. The tangent space of $S L_{n}$ is isomorphic as a vector space to its Lie algebra, which is known to be the $n \times n$ trace zero matrices over $\mathbb{C}$. The Lie algebra of $B$ is the subalgebra of upper triangular matrices with trace zero. Let $G=S L_{n}(\mathbb{C}), \mathfrak{g}=\operatorname{Lie}\left(S L_{n}\right)$ and $\mathfrak{b}=\operatorname{Lie}(B)$. Then the tangent space of $G / B$ at the identity matrix is isomorphic to $\mathfrak{g} / \mathfrak{b}$. Denoting by $E_{i, j}$ the matrix with 1 in the $(i, j)$-entry and 0 's elsewhere, we obtain a basis for $\mathfrak{g} / \mathfrak{b}$ by

$$
\mathfrak{g} / \mathfrak{b}=\operatorname{span}\left\{E_{j, i}: 1 \leq i<j \leq n\right\} .
$$

Observe that there is a natural bijection between the basis elements $\left\{E_{j, i}: i<j\right\}$ and $R:=$ $\left\{t_{i, j}: i<j\right\}$ the set of reflections.

More generally, for any $v \in S_{n}$, the tangent space to $G / B$ at $v$ is given by

$$
T_{v}(G / B)=v(\mathfrak{g} / \mathfrak{b}) v^{-1}=\operatorname{span}\left\{E_{v(j), v(i)}: i<j\right\} .
$$

Why? Because, $G / v B v^{-1}$, is an isomorphic copy of the flag manifold $G / B$ but with respect to the base flag $v_{\bullet}$. Here the flag $v_{\bullet}=v B$ is fixed by the left action of $v B v^{-1}$.

It is an easy exercise to check

$$
\begin{aligned}
& v E_{i j} v^{-1}=E_{v(i), v(j)}, \\
& t_{v(i), v(j)} v=v t_{i j}
\end{aligned}
$$

for any $1 \leq i<j \leq n$. The next theorem gives us an explicit description of a basis of the tangent space of each Schubert variety.

Theorem 3.1. (Lakshmibai-Seshadri 65]) For $v \leq w \in S_{n}$, the tangent space of $X_{w}$ at $v$ is given by

$$
T_{v}\left(X_{w}\right) \cong \operatorname{span}\left\{E_{v(j), v(i)}: i<j, \quad v t_{i j} \leq w\right\},
$$


and hence we obtain

$$
\operatorname{dim} T_{v}\left(X_{w}\right)=\#\left\{(i<j): v t_{i j} \leq w\right\} .
$$

Proof. Recall from the definition of a Lie algebra that $E_{v(j), v(i)} \in T_{v}\left(X_{w}\right)$ is equivalent to $\left(I+\varepsilon E_{v(j), v(i)}\right) v \in X_{w}$ for infinitesimal $\varepsilon>0$ where we can assume $\varepsilon^{2}=0$. Think of $\left(I+\varepsilon E_{v(j), v(i)}\right)$ as a matrix in $G$ acting on the left of the flag $v_{\bullet}$ by moving the flag a little bit in the direction of $E_{v(j), v(i)}$. In particular, $\left(I+\varepsilon E_{v(j), v(i)}\right) v=v+\varepsilon E_{v(j), v(i)} v=$ $v+\varepsilon E_{v(j), i} \in X_{v}$ if and only if $v(i)>v(j)$ which is equivalent to $v t_{i j} \leq v$. Since $v \leq w$ implies $T_{v}\left(X_{v}\right) \subset T_{v}\left(X_{w}\right)$ we see that $E_{v(j), v(i)}$ is in $T_{v}\left(X_{w}\right)$ whenever $v(i)>v(j)$.

On the other hand, if $v(i)<v(j)$ then $v+\varepsilon E_{v(j), i} \in C_{v t_{i j}}$ and so $E_{v(j), v(i)} \in T_{v}\left(X_{w}\right)$ if and only if $v t_{i j} \leq w$. Thus, in either case $E_{v(j), v(i)} \in T_{v}\left(X_{w}\right)$ if and only if $v t_{i j} \leq w$. Thus, $\operatorname{dim} T_{v}\left(X_{w}\right) \geq \#\left\{(i<j): v t_{i j} \leq w\right\}$.

To prove $\operatorname{dim} T_{v}\left(X_{w}\right) \leq \#\left\{(i<j): v t_{i j} \leq w\right\}$, assume there exists coefficients $a_{i, j}$ for $1 \leq i<j \leq n$ such that $v+\varepsilon \sum a_{i, j} E_{v(j), i} \in X_{w}$. Say $v+\varepsilon \sum a_{i, j} E_{v(j), i} \in C_{v^{\prime}}$ for some $v^{\prime} \leq w$. Since $\varepsilon<<1$, none of the minors in $v$ which are nonzero will vanish in $v+\varepsilon \sum a_{i, j} E_{v(j), i}$, so the rank table for $v+\varepsilon \sum a_{i, j} E_{v(j), i}$ dominates the rank table for $v$ in every position. Hence, $v \leq v^{\prime} \leq w$. Thus, for each $a_{i, j} \neq 0$, we have $v+\varepsilon E_{v(j), i} \in X_{w}$ so $\sum a_{i, j} E_{v(j), v(i)}$ is in the span of the independent set of $E_{v(j), v(i)}$ already known to be in $T_{v}\left(X_{w}\right)$.

Corollary 3.2. $X_{w}$ is smooth at $v \in S_{n}$ if and only if

$$
\operatorname{dim} T_{v}\left(X_{w}\right):=\#\left\{(i<j): v t_{i j} \leq w\right\}=l(w)
$$

or equivalently if and only if

$$
\#\left\{(i<j): v<v t_{i j} \leq w\right\}=l(w)-l(v) .
$$

Example 3.3. Consider the case $n=4$. The Schubert variety $X_{4231}$ is not smooth at the point $v=2143$. For all 6 transpositions, $v t_{i j} \leq w$, but $\ell(w)=5$. Also, $6=\#\left\{t_{i j} \leq 4231\right\}=$ $\operatorname{dim} T_{i d}\left(X_{4231}\right)>\ell(4231)=5$. See Figure 2 to verify these statements. Similarly, one can check $X_{3412}$ is not smooth at $v=1324$ and is smooth at all $v^{\prime} \leq w$ such that $v^{\prime} \not \leq v$. It follows that

$$
\begin{aligned}
& \operatorname{Sing}\left(X_{4231}\right)=X_{2143} \\
& \operatorname{Sing}\left(X_{3412}\right)=X_{1324} .
\end{aligned}
$$

Note that 3412 is the reverse of 2143 and 4231 is the reverse of 1324 . All other Schubert varieties $X_{w}$ for $w$ in $S_{4}$ are smooth.

\subsection{Bruhat graphs.}

Definition 3.4. For a permutation $w$, the Bruhat graph for $w$ is a graph whose vertex set is $\left\{v \in S_{n}: v \leq w\right\}=[i d, w]$ and there is an edge between $v$ and $v t_{i j}$ if and only if both $v, v t_{i j} \leq w$.

For example, the Bruhat graph of $w=4321$ is drawn in Figure 3. Observe that the degree of $v$ (i.e. the number of edges connected to $v$ ) in the Bruhat graph for $w$ is $\operatorname{dim} T_{v}\left(X_{w}\right)$.

The Bruhat graph for $w$ has a geometric interpretation: it is the moment graph of the Schubert variety $X_{w}$. Let $T \subset G L_{n}$ be the set of invertible diagonal matrices, then the permutation matrices in $G L_{n} / \tilde{B}$ are exactly the $T$-fixed points.

(i) The permutations in $[i d, w]$ are in bijection with the $T$-fixed points of $X_{w}$.

(ii) If $v, v t_{i j} \leq w$, then the edge between $v$ and $v t_{i j}$ in the Bruhat graph for $w$, is realized as the corresponding curve passing through the flags corresponding to $v$ and $v t_{i j}$

$$
L_{v}=\left\{v+z E_{v(j), i}: z \in \mathbb{C}\right\} \cup\left\{v t_{i j}\right\} \approx \mathbb{P}^{1} .
$$

This curve is $T$-invariant, and pointwise fixed by a torus $T^{\prime} \subset T$ of codimension 1 . 


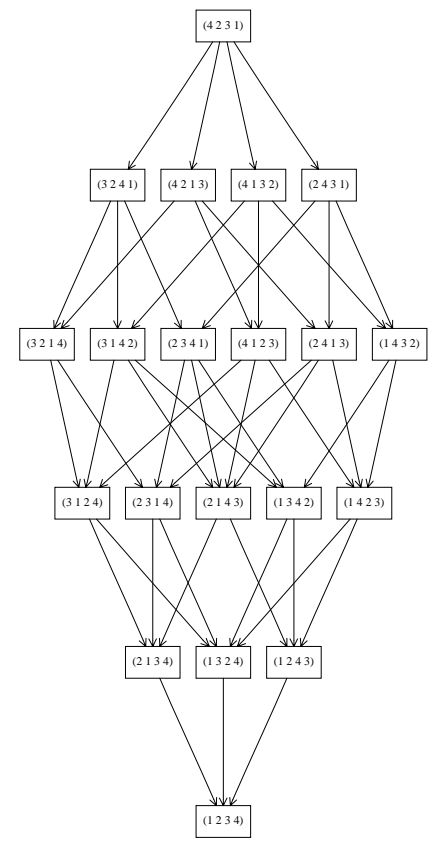

FIgURE 2. The interval $[i d, 4231]$

Schubert varieties are examples of GKM-spaces studied by Goresky-Kottwitz-MacPherson [48] and others. It turns out that much of the T-equivariant topology or geometry of GKM spaces can be described in terms of their moment graph.

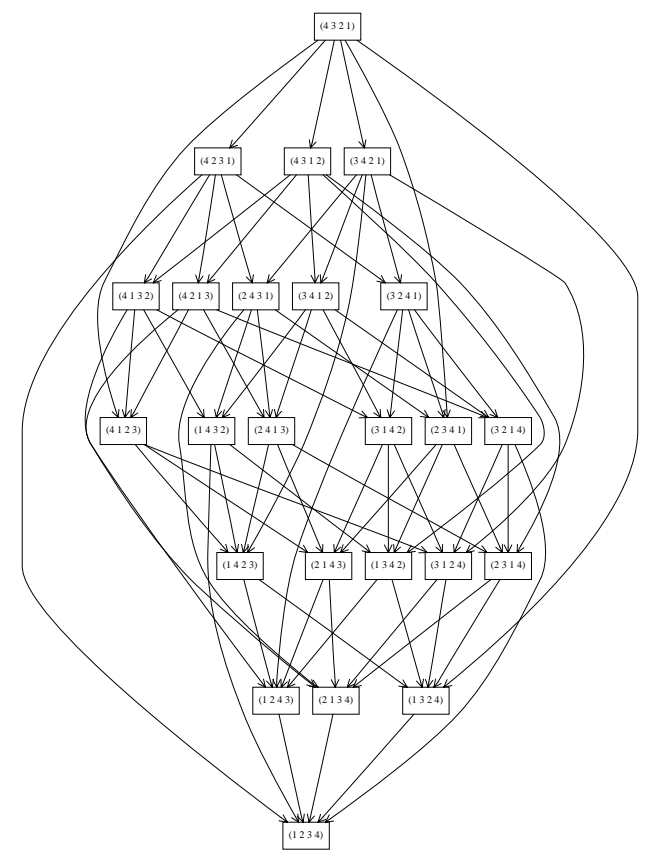

Figure 3. The Bruhat graph of $w=4321$ 
3.3. Lakshmibai-Sandhya Theorem. There exists a simple criterion for characterizing smooth Schubert varieties using permutation pattern avoidance. Pattern avoidance first appeared in work by Knuth [62, Pratt 81 and Tarjan 90 related to computer sorting algorithms in the 1960's and 1970's. Today, many families of permutations are characterized by pattern avoidance or variations on that idea. We discuss one of the key results that brought this technique into the study of Schubert varieties.

Lakshmibai-Sandhya proved the following criterion for the singularity of Schubert varieties in 1990. See also the mutually independent work by Haiman (unpublished), Ryan [86, and Wolper 98 .

Theorem 3.5. (Lakshmibai-Sandhya 64]) $X_{w}$ is singular if and only if $w$ has a subsequence with the same relative order as 3412 or 4231 .

More generally, given any sequence of distinct real numbers $r_{1} \ldots r_{m}$ define $f l\left(r_{1} \ldots r_{m}\right)$ to be the permutation $v \in S_{m}$ such that $r_{i}<r_{j}$ if and only if $v_{i}<v_{j}$. Recall that a permutation is uniquely defined by its inversion set, so this condition uniquely defines v. The $f l$ operator flattens the sequence. Then, a permutation $w=w_{1} w_{2} \ldots w_{n} \in S_{n}$ contains a pattern $v=v_{1} v_{2} \ldots v_{m} \in S_{m}$ for $m<n$ if there exists $i_{1}<i_{2}<\ldots<i_{m}$ such that $f l\left(w_{i_{1}} w_{i_{2}} \ldots w_{i_{m}}\right)=v$. Otherwise, $w$ avoids $v$.

Example 3.6. The permutation $w=625431$ contains the subsequence 6241 which flattens into a 4231-pattern. Hence, $X_{625431}$ is singular. Also, $w=612543$ avoids the patterns 4231 and 3412 which implies that $X_{612543}$ is non-singular.

Let us sketch one approach to proving Theorem 3.5 by applying Theorem 3.1. Say $w$ contains a 3412 or 4231 pattern in positions $i_{1}<i_{2}<i_{3}<i_{4}$. Let $v$ be the permutation obtained from $w$ by rearranging the numbers $w_{i_{1}} w_{i_{2}} w_{i_{3}} w_{i_{4}}$ according to the pattern for the corresponding singular locus in $S_{4}$. Specifically, if $w_{i_{1}} w_{i_{2}} w_{i_{3}} w_{i_{4}}$ is a 4231 then replace $w_{i_{1}} w_{i_{2}} w_{i_{3}} w_{i_{4}}$ by the 2143 pattern $w_{i_{2}} w_{i_{4}} w_{i_{1}} w_{i_{3}}$ in the same positions. If $w_{i_{1}} w_{i_{2}} w_{i_{3}} w_{i_{4}}$ is a 3412 then replace $w_{i_{1}} w_{i_{2}} w_{i_{3}} w_{i_{4}}$ by the 1324 pattern $w_{i_{3}} w_{i_{1}} w_{i_{4}} w_{i_{2}}$ in the same positions. For example, if $w=625431$ and we use the 6241 instance of the pattern 4231, then $v=215634$ which contains a 2143 pattern among the values $1,2,4,6$.

We claim that $X_{w}$ is singular at the point $v$ by construction. The proof proceeds by comparing $\ell(w)-\ell(v)$ with the number of $t_{i j}$ such that $v<v t_{i j} \leq w$. For $i, j \in\left\{i_{1}, i_{2}, i_{3}, i_{4}\right\}$, we know there will be strictly more such transpositions than the length difference in these positions. A key lemma now states that if two permutations $v$ and $w$ agree in position $i$, then $v \leq w$ if and only if $f l\left(v_{1} \ldots \widehat{v}_{i} \ldots v_{n}\right) \leq f l\left(w_{1} \ldots \widehat{w_{i}} \ldots w_{n}\right)$ [6, Lemma 2.1]. This follows from looking at the rank tables of two permutations differing by a transposition. Next, note that $v t_{i j}$ and $w$ differ in at most 6 positions. Thus, by a computer verification on permutations of length 6 one can show that

$$
\#\left\{t_{i j}: v<v t_{i j} \leq w\right\}>\ell(w)-\ell(v) .
$$

In the other direction, assume that $w$ avoids the patterns 4231 and 3412. Lakshmibai and Sandhya show that avoiding these patterns is equivalent to an equidimensionality property of certain projections which implies smoothness.

Haiman's proof also contained the following enumerative formula as a corollary. Since his paper was never published, it wasn't until 2007 that this result had a proof in the literature due to Bousquet-Mélou and Butler.

Corollary 3.7. [19] There is a closed form for the generating function for the sequence $v_{n}$ counting the number of smooth Schubert varieties for $w \in S_{n}$ :

$$
\begin{aligned}
V(t) & =\frac{1-5 t+3 t^{2}+t^{2} \sqrt{1-4 t}}{1-6 t+8 t^{2}-4 t^{3}} \\
& =1+t+2 t^{2}+6 t^{3}+22 t^{4}+88 t^{5}+366 t^{6}+1552 t^{7}+6652 t^{8}+O\left(t^{9}\right) .
\end{aligned}
$$


Note that by the Lakshmibai-Sandhya theorem, testing for smoothness of Schubert varieties can be done naively in polynomial time, $O\left(n^{4}\right)$, based on the characterization of avoiding 3412 and 4231. As we pointed out in the introduction, the Guillemot-Marx 51] construction leads to a linear time algorithm in $n$ for testing if a permutation in $S_{n}$ contains either a 3412 or 4231 pattern.

Historically, there were some incremental results leading up to the linear time algorithm to detect pattern avoidance by Guillemot and Marx. These other algorithms might still have useful applications, so we mention a couple of them here. In 71, Madras and Liu study the 4231-avoiding permutations. They point out that using Knuth's original characterization of stack-sortable permutations in linear time, one can find a 4231 pattern in $O\left(n^{2}\right)$ time. In fact, Albert-Aldred-Atkinson-Holton show that every length 4 pattern can be detected in $O(n \log n)$ time [3.

\section{10 Pattern Avoidance Properties}

In this section, we exhibit the ubiquity of pattern avoidance as a tool to characterize important properties in Schubert geometry and related areas. We give 10 distinct properties which are characterized by pattern avoidance. Each property will have a description in terms of avoiding certain patterns. Often these permutation families have other distinguishing features as well.

The first family of permutations defined by pattern avoidance is the 3412 - and 4231avoiding permutations appearing in the Lakshmibai-Sandhya Theorem. It is a family rich in structure. For the record, we state all the properties equivalently characterized by these two patterns. The history, citations, and some definitions follow the statement.

Pattern Avoidance Property 1. The following are equivalent for $w \in S_{n}$.

(1) The one-line notation for $w$ avoids 3412 and 4231.

(2) $X_{w}$ is smooth.

(3) $\ell(w)=\#\left\{t_{i j} \leq w\right\}$.

(4) The Bruhat graph for $w$ is regular and every vertex has degree $\ell(w)$.

(5) The Poincaré polynomial for $w, P_{w}(t)=\sum_{v \leq w} t^{l(v)}$ is palindromic.

(6) The Poincaré polynomial for $w$ factors as

$$
P_{w}(t)=\prod_{i=1}^{k}\left(1+t+t^{2}+\cdots+t^{e_{i}}\right)
$$

for some positive integers $\left\{e_{1}, e_{2}, \ldots, e_{k}\right\}$ such that $\ell(w)=\sum e_{i}$.

(7) The Poincaré polynomial $P_{w}(t)$ is equal to the generating function $R_{w}(t)$ for the number of regions $r$ in the complement of the inversion hyperplane arrangement weighted by the distance of each region to the fundamental region. In symbols,

$$
R_{w}(t)=\sum_{r} t^{d(r)}=\sum_{v \leq w} t^{l(v)}=P_{w}(t) .
$$

Here, $d(r)$ is the number of hyperplanes crossed in a walk starting at the fundamental region and going to the region $r$.

(8) The inversion arrangement for $w$ is free and the number of chambers of the arrangement is equal to the size of the Bruhat interval $[i d, w]$.

(9) The Kazhdan-Lusztig polynomial $P_{x, w}(t)=1$ for all $x \leq w$.

(10) The Kazhdan-Lusztig polynomial $P_{i d, w}(t)=1$.

We have already discussed the equivalence of the first three items. Items (4), (5), and (10) are due to Carrell and Peterson [27]. Note, Carrell is the sole author on the paper cited, but he always acknowledges Peterson as a collaborator on this work so we give them both 
credit. The term palindromic refers to the sequence of coefficients of the polynomial, so the coefficient of $t^{i}$ equals the coefficient of $t^{\ell(w)-i}$ in a palindromic Poincaré polynomial.

Item (6) about factoring Poincaré polynomials is due to Gasharov 44. This factorization implies that the geometry of smooth Schubert varieties has particularly nice structure in terms of iterated fiber bundles over Grassmannians [46, 84, 84, 86, 98.

Example 4.1. The permutation $w=4321$ avoids the patterns 3412 and 4231 . It has a palindromic Poincaré polynomial that also factors nicely,

$$
\begin{aligned}
P_{4321}(t) & =(1+t)\left(1+t+t^{2}\right)\left(1+t+t^{2}+t^{3}\right) \\
& =1+3 t+5 t^{2}+6 t^{3}+5 t^{4}+3 t^{5}+t^{6} .
\end{aligned}
$$

Example 4.2. The permutation 3412 is one of the two cases in $S_{4}$ where the Poincaré polynomial does not have the nice factorization, nor the palindromic property. Here

$$
P_{3412}(t)=1+3 t+5 t^{2}+4 t^{3}+t^{4} .
$$

Item (7) about the inversion hyperplane arrangement is due to Oh-Postnikov-Yoo [78]. This arrangement is given by the collection of hyperplanes defined by $x_{i}-x_{j}=0$ for all $i<j$ such that $w(i)>w(j)$. This generalizes the notion of the Coxeter arrangement of type $A_{n-1}$ given by all the hyperplanes $x_{i}-x_{j}=0$ for all $i<j$, so it is the inversion arrangement for $w_{0}$. The Coxeter arrangement has $n$ ! regions corresponding to all the permutations. In this case, the statistic $d(w)$ equals $\ell(w)$. Note no explicit bijective proof of Item (7) is known. The inversion arrangement comes up again in Property 5 below.

Item (8) is due to Slofstra [87]. Here a central hyperplane arrangement in a Euclidean space $V$ is said to be free if the module of derivations of the complexified arrangement is free as a module over the polynomial ring $\mathbb{C}\left[V_{\mathbb{C}}\right]$. We refer the reader to this paper for more background. Note it also gives an algebraic interpretation for the generalized exponents $e_{1}, e_{2}, \ldots, e_{k}$ in terms of degrees of a homogeneous basis for the module of derivations.

Items (9) and (10) concern the Kazhdan-Lusztig polynomials [59]. These polynomials play an important role in the study of the singularities of Schubert varieties and in representation theory. We recall the definitions here, highlight some important developments, and refer the reader to the textbooks by Humphreys [54] and Björner-Brenti [17] for more details.

The Hecke algebra $\mathcal{H}$ associated with $S_{n}$ is an algebra over $\mathbb{Z}\left[q^{\frac{1}{2}}, q^{\frac{-1}{2}}\right]$ generated by $\left\{T_{i}: 1 \leq\right.$ $i \leq n-1\}$ with the relations

(1) $\left(T_{i}\right)^{2}=(q-1) T_{i}+q$

(2) $T_{i} T_{j}=T_{j} T_{i} \quad$ if $|i-j|>1$,

(3) $T_{i} T_{i+1} T_{i}=T_{i+1} T_{i} T_{i+1}$ for all $1 \leq i<n$.

This definition is patterned after the definition of the symmetric group $S_{n}$ written in terms of its generating set of adjacent transpositions and their relations. In fact, if we take the specialization $q=1$, then the resulting algebra is the group algebra of $S_{n}$. The relations $(2)$ and (3) are called the braid relations. The braid relations imply that $T_{w}=T_{i_{1}} T_{i_{2}} \cdots T_{i_{p}}$ is well defined for any reduced expression $w=s_{i_{1}} s_{i_{2}} \ldots s_{i_{p}}$. We will use the notation $T_{i d}=1 \in \mathcal{H}$ for the empty product of generators.

An easy observation is that $\left\{T_{w}: w \in S_{n}\right\}$ is a linear basis for $\mathcal{H}$ over $\mathbb{Z}\left[q^{\frac{1}{2}}, q^{\frac{-1}{2}}\right]$. One can also observe that the $T_{w}$ 's are invertible over $\mathbb{Z}\left[q, q^{-1}\right]$ which can be see as follows. First check that $\left(T_{i}\right)^{-1}=q^{-1} T_{i}-\left(1-q^{-1}\right)$ by multiplying by $T_{i}$ and using the stated relations. Then, we have $\left(T_{w}\right)^{-1}=\left(T_{i_{p}}\right)^{-1} \ldots\left(T_{i_{1}}\right)^{-1}$ for a reduced expression $w=s_{i_{1}} s_{i_{2}} \ldots s_{i_{p}}$.

Next, let us review the Kazhdan-Lusztig involution. Consider the $\mathbb{Z}$-linear transformation $i: \mathcal{H} \rightarrow \mathcal{H}$ sending $T_{w}$ to $\left(T_{w^{-1}}\right)^{-1}$ and $q$ to $q^{-1}$, respectively.

Theorem 4.3. (Kazhdan-Lusztig [59]) There exists a unique basis $\left\{C_{w}^{\prime}: w \in S_{n}\right\}$ for the Hecke algebra $\mathcal{H}$ over $\mathbb{Z}\left[q^{\frac{1}{2}}, q^{\frac{-1}{2}}\right]$ such that 
(i) $i\left(C_{w}^{\prime}\right)=C_{w}^{\prime}$.

(ii) The change of basis matrix from $\left\{C_{w}^{\prime}\right\}$ to $\left\{T_{w}\right\}$ is upper triangular when the elements of $S_{n}$ are listed in a total order respecting Bruhat order, and the expansion coefficients $P_{x, w}(q)$ in

$$
C_{w}^{\prime}=q^{-\frac{1}{2} \ell(w)} \sum_{x \leq w} P_{x, w}(q) T_{x}
$$

have the properties $P_{w, w}=1$ and for all $x<w, P_{x, w}(q) \in \mathbb{Z}[q]$ with degree at most

$$
\frac{\ell(w)-\ell(x)-1}{2} .
$$

The basis $\left\{C_{w}^{\prime}: w \in S_{n}\right\}$ is called the Kazhdan-Lusztig basis for $\mathcal{H}$, and $P_{x, w}(q)$ is the Kazhdan-Lusztig polynomial for $x, w \in S_{n}$. This theorem easily generalizes to all Coxeter groups for the reader familiar with that topic.

Example 4.4. We exhibit some computations with the Kazhdan-Lusztig basis indexed by permutations with the aid of Theorem 4.3. First, it is easy to see

$$
C_{s_{i}}^{\prime}=q^{-\frac{1}{2}}\left(1+T_{i}\right)=q^{\frac{1}{2}}\left(1+T_{i}^{-1}\right) .
$$

Then, for $i \neq j$, the computation

$$
C_{s_{i}}^{\prime} C_{s_{j}}^{\prime}=q^{-1}\left(1+T_{i}\right)\left(1+T_{j}\right)=q^{-1}\left(1+T_{i}+T_{j}+T_{i} T_{j}\right)
$$

shows that $C_{s_{i} s_{j}}^{\prime}=C_{s_{i}}^{\prime} C_{s_{j}}^{\prime}$ for $i \neq j$. Also, in the computation

$$
\begin{aligned}
C_{s_{1}}^{\prime} C_{s_{2}}^{\prime} C_{s_{1}}^{\prime} & =q^{-\frac{3}{2}}\left(1+T_{1}\right)\left(1+T_{2}\right)\left(1+T_{1}\right) \\
& =q^{-\frac{3}{2}}\left(1+2 T_{1}+T_{2}+T_{1} T_{2}+T_{2} T_{1}+T_{1}^{2}+T_{1} T_{2} T_{1}\right) \\
& =q^{-\frac{3}{2}}\left(1+2 T_{1}+T_{2}+T_{1} T_{2}+T_{2} T_{1}+\left((q-1) T_{1}+q\right)+T_{1} T_{2} T_{1}\right),
\end{aligned}
$$

one notices that $q T_{1}+q$ which comes from $T_{1}^{2}$ should not appear for $C_{s_{1} s_{2} s_{1}}^{\prime}$ because the degree of the polynomial coefficient of $T_{1}$ and $T_{i d}$ are too large. We need a correction term. Since $C_{s_{i}}^{\prime}=q^{-\frac{1}{2}}\left(1+T_{i}\right)$ one can check that $C_{s_{1} s_{2} s_{1}}^{\prime}=C_{s_{1}}^{\prime} C_{s_{2}}^{\prime} C_{s_{1}}^{\prime}-C_{s_{1}}^{\prime}$ by Theorem 4.3

Example 4.5. If $i_{1}, \cdots, i_{k} \in[n-1]$ are distinct, then one can check that

$$
C_{s_{i_{1}} \cdots s_{i_{k}}}^{\prime}=C_{s_{i_{1}}}^{\prime} \cdots C_{s_{i_{k}}}^{\prime} \text {. }
$$

More generally, a permutation $w \in S_{n}$ is called Deodhar if $C_{w}^{\prime}=C_{s_{i_{1}}}^{\prime} C_{s_{i_{2}}}^{\prime} \cdots C_{s_{i_{p}}}^{\prime}$ for some reduced expression $w=s_{i_{1}} s_{i_{2}} \cdots s_{i_{p}}$. We will return to the Deodhar permutations in Property 6.

Example 4.6. The Kazhdan-Lusztig polynomials $P_{\mathrm{id}, w}$ for $w \in S_{5}$ are completely determined from the following table and the fact that $P_{\mathrm{id}, w}=1$ if and only if $w$ is 3412 and 4231 avoiding.

\begin{tabular}{|ccc|c|}
\hline \multicolumn{3}{|c|}{$w$} & $P_{\mathrm{id}, w}$ \\
\hline$(14523)$ & $(15342)$ & $(24513)$ & \\
$(25341)$ & $(34125)$ & $(34152)$ & \\
$(35124)$ & $(35142)$ & $(35241)$ & \\
$(35412)$ & $(41523)$ & $(42315)$ & \\
$(42351)$ & $(42513)$ & $(42531)$ & $q+1$ \\
$(43512)$ & $(45132)$ & $(45213)$ & \\
$(51342)$ & $(52314)$ & $(52413)$ & \\
$(52431)$ & $(53142)$ & $(53241)$ & \\
$(53421)$ & $(54231)$ & & $2 q+1$ \\
\hline$(34512)$ & $(45123)$ & $q^{2}+1$ \\
\hline$(45231)$ & $(53412)$ & $2 q+1$ \\
\hline \multicolumn{3}{|c|}{$(52341)$} \\
\hline
\end{tabular}


The reader might notice that all coefficients of Kazhdan-Lusztig polynomials shown so far are non-negative integers. In their 1979 paper, this property was stated as a conjecture for all Kazhdan-Lusztig polynomials. In 1980, Kazhdan and Lusztig proved their own conjecture using intersection homology as introduced by Goresky and MacPherson in 1974, see [49] as a good starting point for that theory.

Theorem 4.7. (Kazhdan-Lusztig [60]) If $W$ is a Weyl group or affine Weyl group then

$$
P_{x, w}(q)=\sum \operatorname{dim} \mathcal{I} \mathcal{H}_{x}^{i}\left(X_{w}\right) q^{i} .
$$

Corollary 4.8. The coefficients of $P_{x, w}(q)$ are non-negative integers with constant term 1.

The big news in Kazhdan-Lusztig theory is the recent proof that all Kazhdan-Lusztig polynomials for all Coxeter groups have non-negative integer coefficients. This proof is due to Elias and Williamson 40. They give an algebraic structure (Soergel bimodules) which plays the same role as intersection homology of Schubert varieties in the original proof.

As stated in Property 1, Kazhdan-Lusztig polynomials can be used to determine smoothness of Schubert varieties (in type A). There are several other interesting properties of Kazhdan-Lusztig polynomials that have emerged since they were defined in 1979. We cover some of them here and recommend the Wikipedia page [97] for a very nice survey.

(1) In 1981, Beilinson-Bernstein, and independently Brylinski-Kashiwara, proved another important conjecture due to Kazhdan and Lusztig. They showed that the multiplicities which appear when expressing the formal character of a Verma module in terms of the formal character for the corresponding simple highest weight module are determined by evaluating Kazhdan-Lusztig polynomials at $q=1$ ([5, 25]).

(2) The coefficients of Kazhdan-Lusztig polynomials are increasing as one goes down in Bruhat order, while keeping the second index fixed. Specifically, if $x \leq y \leq w$, then $\operatorname{coef}_{q^{k}} P_{x, w}(q) \geq \operatorname{coef}_{q^{k}} P_{y, w}(q)$. This monotonicity property was first published in 1988 by Ron Irving 56. Irving's proof is based on the socle filtration of a Verma module. In 2001, Braden and MacPherson gave a different proof using intersection homology [20, Cor. 3.7].

(3) Every polynomial with constant term 1 and nonnegative integer coefficients is the Kazhdan-Lusztig polynomial of some pair of permutations. This is due to Patrick Polo, published in 1999 [80. He gives an explicit construction of the pair of permutations for a given polynomial. This was a surprising result because from the small data that we can compute, say for $n \leq 8$, the polynomials seem quite special. They must get increasingly complex as $n$ grows.

(4) Let $\mu(x, w)$ be the coefficient of $q^{\frac{\ell(w)-\ell(x)-1}{2}}$ in $P_{x, w}$. Note, $\mu(x, w)$ can be 0 . For $x, w \in S_{9}, \mu(x, w) \in\{0,1\}$. MacLarnen and Warrington found an example in $S_{10}$ where $\mu(x, w)=5$ [75]. Prior to their publication in 2003, this was referred to as the "0-1 Conjecture for Kazhdan-Lusztig polynomials." This again demonstrates the increasing complexity as $n$ grows. The reader might be wondering how anyone could have believed the 0-1 Conjecture after seeing Polo's theorem in (3). However, Polo's theorem does not contradict the 0-1 Conjecture because in his construction the length difference between $w$ and $x$ is large enough that the leading term in $P_{x, w}(q)$ is typically not the $\mu$-coefficient.

(5) There exists a formula for $P_{x, w}(q)$ which only depends on the abstract interval $[i d, w]$ in Bruhat order. See the work of du Cloux (2003) [35], Brenti (2004) [21] and BrentiCaselli-Marietti (2006) [22].

There are two interesting but difficult open problems in Kazhdan-Lusztig theory. There are many partial answers to these questions in the literature, but we don't know of a complete source at this time. Perhaps there is a need for someone to start a wiki page. 
Question 1. (Lusztig) Can one compute $P_{x, w}(q)$ using only the abstract poset given by the interval $[x, w]$ in Bruhat order? In other words, $P_{u, v}(q)=P_{x, y}$ whenever $[u, v]$ and $[x, y]$ are isomorphic as posets.

Question 2. Can one compute the coefficients of $P_{x, w}(q)$ by counting combinatorially defined objects?

The next pattern property connects the 3412 and 4231 patterns to the determination of the singular locus of a Schubert variety. Recall from Section 3 , the singular locus of a Schubert variety $X_{w}$ is a union of Schubert varieties $X_{v}$ with $v<w$. Thus to determine the irreducible components of the singular locus, we just need to give the maximal permutations $v<w$ such that $v$ determines a singular point in $X_{w}$.

Pattern Avoidance Property 2. (Billey-Warrington, Manivel, Kassel-Lascoux-Reutenauer, and Cortez [16, 72, 58, 32]) $X_{v}$ is an irreducible component of the singular locus of $X_{w}$ if and only if

$$
v=w \cdot(1 \text {-cycle permutation) }
$$

corresponding to a 4231 or 3412 or 45312 pattern from Figure 4 such that the shaded region contains no additional 1's except in the 45312 case where they must appear in the central region in decreasing order. Here $\circ$ 's denote 1's in $w$, •'s denote 1's in $v$.

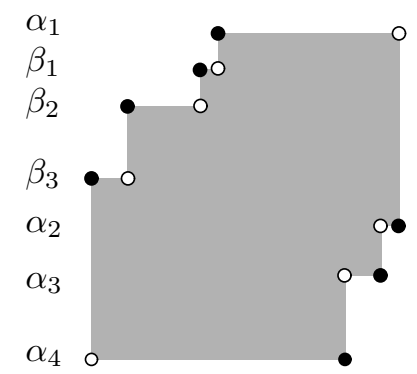

(1)

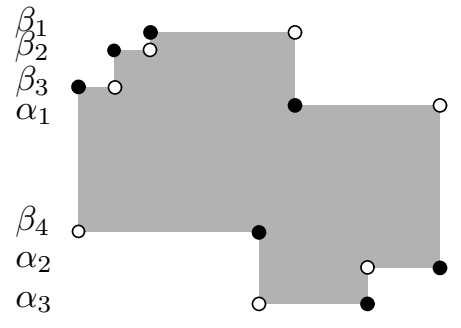

$(2)$

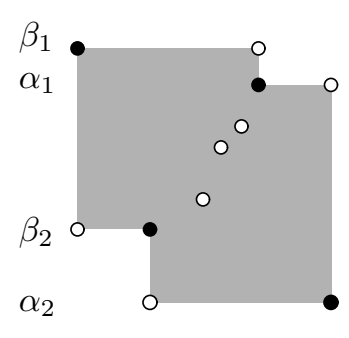

(3)

Figure 4. Patterns for the singular locus of $X_{w}$ in the 4231, 3412, and 45312 cases respectively.

This result was found around 2000 by 7 authors in 4 papers, plus Gasharov proved on direction of the conjecture [45] around the same time. It must have been ripe for discovery. It refined and proved a conjecture due to Lakshmibai and Sandhya 64. For the sake of history, we note that the authors of [16] were the first to report this result to Lakshmibai.

Corollary 4.9. The codimension of the singular locus of a Schubert variety $X_{w}$ is at least 3 for any $w \in S_{n}$.

The corollary is in fact true for all simply laced types. However, it is not true in type $B_{n}$. The codimension of the singular locus of a Schubert variety in that case can be 2 .

Inspired by the Lakshmibai-Sandhya Theorem and the construction of the singular locus of a Schubert variety in Property 2, Woo and Yong [102] defined the notion of interval pattern avoidance. Given permutations $u<v \in S_{m}$ and $x<y \in S_{n}$ for $m<m$, say [u,v] interval pattern embeds into $[x, y]$ provided

(1) There exist indices $1 \leq i_{1}<i_{2}<\ldots<i_{m} \leq n$ such that $\mathrm{fl}\left(x_{i_{1}}, \ldots, x_{i_{m}}\right)=u$ and $\mathrm{fl}\left(y_{i_{1}}, \ldots, y_{i_{m}}\right)=v$ respectively.

(2) The permutations $x, y$ agree in all positions other than $1 \leq i_{1}<i_{2}<\ldots<i_{m} \leq n$.

(3) The Bruhat intervals $[u, v]$ and $[x, y]$ are isomorphic as posets. 
In fact, if $x, y$ agree everywhere outside of the indices $1 \leq i_{1}<i_{2}<\ldots<i_{m} \leq n$ and $u=\mathrm{fl}\left(x_{i_{1}}, \ldots, x_{i_{m}}\right), v=f l\left(y_{i_{1}}, \ldots, y_{i_{m}}\right)$ then $[u, v]$ interval embeds in $[x, y]$ if and only if $\ell(v)-\ell(u)=\ell(y)-\ell(x)$ [102, Lemma 2.1]. Furthermore, for all $w \in S_{n}$ such that $x<w<y$, then $w$ agrees everywhere with $y$ outside of the sequence and $\left[f l\left(w_{i_{1}}, \ldots, w_{i_{m}}\right), v\right]$ also interval embeds in $[x, y]$ [102, Lemma 2.4].

Observe that the condition from Figure 4 that the shaded region have no additional 1's in the permutation matrices implies that the length $l(w)-l(v)$ is equal to the corresponding length drop in each of the 4231,3412 or 45312 cases. Thus, the maximal singular locus of a Schubert variety is determined by interval pattern conditions.

Another example of the power of interval pattern embeddings is the following result supporting Question 1. More examples will follow, but the reader is encouraged to see 102 for more details.

Theorem 4.10. [102, Cor. 6.3] Suppose $[u, v] \subset S_{m}$ interval pattern embeds into $[x, y] \in S_{n}$, then the Kazhdan-Lusztig polynomials $P_{u, v}(q)$ and $P_{x, y}(q)$ are equal.

Next, recall by a theorem due to Zariski that a variety $X$ is smooth if and only if the local ring at every point is regular. A variety $X$ is factorial at a point if the local ring at that point is a unique factorization domain. Note that a smooth variety is factorial at every point since any regular local ring is a unique factorization domain. The following property was conjectured by Woo-Yong and proved by Bousquet-Mélou and Butler in 2007.

Pattern Avoidance Property 3. (Bousquet-Mélou-Butler [19]) Let $w \in S_{n}$, then the following are equivalent.

(1) The Schubert variety $X_{w}$ is factorial at every point.

(2) The permutation $w$ avoids 4231 and $3 \underline{412}$ where $3 \underline{412}$ means that the 4 and 1 must be adjacent in the one-line notation for $w$.

(3) The permutation $w$ avoids 4231, and for every $v<w$ differing in exactly 4 positions, the interval $[v, w]$ is not isomorphic to $[3142,3412]$. Thus, one says $w$ interval avoids $[3142,3412]$.

Compare the generating function below with Corollary 3.7 which is the generating function for the number of smooth Schubert varieties in $\mathcal{F} l_{n}$.

Theorem 4.11. 19] There is a closed form for the generating function for the sequence $f_{n}$ counting the factorial Schubert varieties for $w \in S_{n}$ :

$$
\begin{aligned}
F(t) & =\frac{(1-t)\left(1-4 t-2 t^{2}\right)-(1-5 t) \sqrt{(1-4 t)}}{2\left(1-5 t+2 t^{2}-t^{3}\right)} \\
& =t+2 t^{2}+6 t^{3}+22 t^{4}+89 t^{5}+379 t^{6}+1661 t^{7}+7405 t^{8}+\ldots
\end{aligned}
$$

Note, the term $\sqrt{1-4 t}$ appears in both (3) and (4). This term is familiar in combinatorics because it also appears in the generating function for the Catalan numbers, $c_{n}=\frac{1}{n+1}\left(\begin{array}{c}2 n \\ n\end{array}\right)$. In particular, as a power series $\sqrt{1-4 t}=1+\sum_{n>1} \frac{-2}{n}\left(\begin{array}{c}2 n-2 \\ n-1\end{array}\right) t^{n}$ by Newton's generalized binomial theorem. Thus, the generating function for the Catalan numbers is

$$
\sum_{n \geq 1} c_{n} t^{n}=\frac{1-\sqrt{1-4 t}}{2 t} .
$$

There exists a simple criterion for characterizing Gorenstein Schubert varieties using modified pattern avoidance. Recall that a variety $X$ is Gorenstein if it is Cohen-Macaulay and its canonical sheaf is a line bundle. Woo and Yong characterized the Gorenstein condition by using pattern avoidance. 
Pattern Avoidance Property 4. (Woo-Yong 101]) A Schubert variety $X_{w}$ is Gorenstein if and only if the following two conditions are satisfied:

(i) $w$ avoids 35142 and 42513 with Bruhat restrictions $\left\{t_{15}, t_{23}\right\}$ and $\left\{t_{15}, t_{34}\right\}$, and

(ii) for each descent $d$ in $w$, the associated partition $\lambda_{d}(w)$ has all of its inner corners on the same antidiagonal.

Later, Woo-Yong [102, Thm. 6.6] also gave a characterization of Gorenstein Schubert varieties in terms of an interval pattern avoidance using an infinite number of intervals.

We note that in the paper [101, the theorem states that $w$ should avoid 31542 and 24153 which is twisted by $w_{0}$ from the permutations written above. The difference is that they are labeling Schubert varieties in such a way that the codimension of $X_{w}$ is $\ell(w)$ which works better for computing products of Schubert classes.

The proof of this result due to Woo and Yong relates the Gorenstein property to Schubert classes for the flag manifold and Monk's formula. Since the topic of the conference in Osaka is "Schubert Calculus", we outline this proof to show the logical relationship. The steps are due to Woo and Yong unless otherwise mentioned.

Sketch of proof.

Step 1: Schubert varieties are all Cohen-Macaulay. (Ramanathan, 1985)

Step 2: Testing if $X_{w}$ is Gorenstein reduces to a comparison using the Weil divisor class group and the Cartier class group for $X_{w}$. (Brion, Knutson, Kumar)

Step 3: The Weil divisor class group is generated by the set of all $\left[X_{v}\right] \in H^{*}(G / B)$ such that $v<w$ and $\ell(v)=\ell(w)-1$. In this case we say $w$ covers $v$ in Bruhat order, denoted $v \triangleleft w$. If $v \triangleleft w$, then $w=v t_{i j}$ but $t_{i, j}$ does not need to be an adjacent transposition.

Step 4: The Cartier class group is generated by $\left[X_{w_{0} s_{i}}\right]\left[X_{w}\right]$ and

$$
\left[X_{w_{0} s_{i}}\right]\left[X_{w}\right]=\sum\left[X_{v}\right]
$$

summed over all $v=w t_{a b}: a \leq i<b, \ell(v)=\ell(w)-1$ by Monk's formula.

Step 5: The Schubert variety $X_{w}$ is Gorenstein if and only if there exists an integral solution $\left(\alpha_{1}, \ldots, \alpha_{n-1}\right)$ to

$$
\sum_{i=1}^{n-1} \alpha_{i}\left[X_{w_{0} s_{i}}\right]\left[X_{w}\right]=\sum_{v \triangleleft w}\left[X_{v}\right] .
$$

For the details of the proof, see [101].

A Schubert variety $X_{w}\left(E_{\bullet}\right)$ is defined by inclusions if it can be described as the set of all flags $F_{\bullet}$ where $F_{i} \subset E_{j}$ or $E_{i} \subset F_{j}$ for some collection of pairs $i, j$.

Pattern Avoidance Property 5. (Gasharov-Reiner [46]) A Schubert variety $X_{w}$ is defined by inclusions if and only if $w$ avoids 4231, 35142, 42513, 351624.

The four patterns appearing in this property have two other interesting and unexpected connections found using Tenner's Database of Permutation Pattern Avoidance.

Theorem 4.12. (Hultman-Linusson-Shareshian-Sjöstrand [53]) The number of regions in the inversion arrangement for $w$ is at most the number of elements below $w$ in Bruhat order. The two quantities are equal if and only if $w$ avoids 4231, 35142, 42513, 351624.

Given a subset $S$ of $[n] \times[n]$, let $\operatorname{mat}_{q}(n, S, r)$ be the number of $n \times n$ matrices over $\mathbb{F}_{q}$ with rank $r$, none of whose nonzero entries lie in $S$. For example, if $S=\emptyset$, then

$$
\operatorname{mat}_{q}(n, \emptyset, n)=q^{\left(\begin{array}{c}
n \\
2
\end{array}\right)}(q-1)^{n} \prod_{i=1}^{n}\left(1+q+\ldots+q^{i-1}\right)=q^{\left(\begin{array}{c}
n \\
2
\end{array}\right)}(q-1)^{n} P_{w_{0}}(q)
$$

where $w_{0}$ is the longest element of $S_{n}$ and $P_{w_{0}}(q)$ is the Poincaré polynomial for $X_{w_{0}}$. 
Theorem 4.13. (Lewis-Morales [68]) Fix a permutation $w$ in $S_{n}$, and let $D(w)$ be its permutation diagram. We have that

$$
\operatorname{mat}_{q}(n, D(w), n) /(q-1)^{n}=q^{n(n-1)-i n v(w)} P_{w w_{0}}\left(q^{-1}\right)
$$

If and only if $w$ avoids 1324, 24153, 31524, and 426153 (the reverses of the patterns in Property 5).

The theorem above was originally part of a more general conjecture by Klein-Lewis-Morales. We state the part that is still open.

Conjecture 4.1. (Klein-Lewis-Morales 61, Conj. 5.1 and Conj 6.6]) Using the notation above, mat $_{q}(n, D(w), n) /(q-1)^{n}$ is a polynomial function of $q$ which is coefficient-wise less than or equal to $q^{\left(\begin{array}{c}n \\ 2\end{array}\right)-i n v(w)} P_{w}(q)$.

Recently, Albert and Brignall have shown that the enumeration of Schubert varieties defined by inclusions has a nice generating function and recurrence relation. Once again, it is interesting to compare this formula with (3) and (4).

Theorem 4.14. (Albert-Brignall 4]) Let $f(n)$ be the number of permutations in $S_{n}$ which avoid 4231, 35142, 42513, and 351624. Then, we have the generating function

$$
\sum f(n) t^{n}=\frac{1-3 t-2 t^{2}-\left(1-t-2 t^{2}\right) \sqrt{1-4 t}}{1-3 t-\left(1-t+2 t^{2}\right) \sqrt{1-4 t}} .
$$

Gasharov-Reiner give a nice description of the cohomology rings of Schubert varieties defined by inclusions. This result has been extended by Reiner-Woo-Yong in a beautiful way which relates to Fulton's essential set which is a subset of the diagram of a permutation. In order to describe it here, let us first recall Carrell's result on the cohomology of Schubert varieties.

Theorem 4.15. (Carrell [26]) $H^{*}\left(X_{w}\right) \approx H^{*}(G / B) / I_{w}$ where $I_{w}$ is generated by all $\left[X_{v}\right]$ such that $v \not \leq w$.

A permutation $x$ is called Grassmannian if $x$ has at most 1 descent. Also, $x$ is bigrassmannian if both $x$ and $x^{-1}$ are Grassmannian. We denote by Des $(x)$ the set of descents in $x$. In 1992, Akyildiz-Lascoux-Pragacz gave a description of the ideal $I_{w}$ which was then further refined by Reiner-Woo-Yong.

Theorem 4.16. (Akyildiz-Lascoux-Pragacz 2]) $I_{w}$ is generated by the set of all $\left[X_{v}\right]$ such that $v \leq \leq w$ and $v$ is Grassmannian.

Following [83, for a permutation $w$, let $E(w)$ be the set of permutations which are minimal elements in Bruhat order in the complement of the interval $[i d, w]$. The set $E(w)$ is called the essential set of $w$. Clearly, this notion of essential set generalizes to all Coxeter groups.

Theorem 4.17. (Lascoux-Schützenberger and Geck-Kim [4]) The elements in E(w) are bigrassmannian.

Theorem 4.18. (Reiner-Woo-Yong [83]) There exists a bijection between E(w) and Fulton's essential set which is defined as the cells in the diagram of the permutation $D(w)$ which have no cell directly to their right or below.

Theorem 4.19. (Reiner-Woo-Yong [83]) $I_{w}$ is generated by the set of all $\left[X_{v}\right]$ such that $v \not \leq w, v$ is Grassmannian and there exists some bigrassmannian $x \in E(w)$ such $x \leq v$ and $\operatorname{Des}(x)=\operatorname{Des}(v)$.

Reiner-Woo-Yong point out that this generating set for $I_{w}$ is still not minimal in general. This leads to some interesting open questions. 
Question 3. Find a minimal set of generators for $I_{w}$ for all $w \in S_{n}$. (See [83]).

Question 4. What is the relationship between $E(w)$ and the defining equations for Schubert varieties in other types?

The next property relates the Bott-Samelson resolution for a singular Schubert variety and the Kazhdan-Lusztig basis elements to pattern avoidance. A resolution of a singular variety is called a small resolution if for every $r>0$, the space of points of $X$ where the fiber over the point in the resolution has dimension $r$ is of codimension greater than $2 r$. In words, the singular points where the resolution has to blow up the dimension a lot are rare in a small resolution. One reason that people care about small resolutions is that the intersection homology of a variety is just the homology of a small resolution of the variety.

Pattern Avoidance Property 6. (Deodhar 34, Billey-Warrington 12]) The following are equivalent.

(1) $C_{w}^{\prime}=C_{s_{i_{1}}}^{\prime} C_{s_{i_{2}}}^{\prime} \cdots C_{s_{i_{p}}}^{\prime}$ for some (or any) reduced expression $w=s_{i_{1}} s_{i_{2}} \cdots s_{i_{p}}$.

(2) The Bott-Samelson resolution of $X_{w}$ is small.

(3) $\sum_{v \leq w} t^{l(v)} P_{v, w}(t)=(1+t)^{l(w)}$.

(4) For each $v \leq w$, the Kazhdan-Lusztig polynomial can be written as

$$
P_{v, w}(t)=\sum_{\sigma \in E(v, w)} t^{\operatorname{defect}(\sigma)} .
$$

(5) $w$ is 321-hexagon avoiding, that is, $w$ avoids

$$
321,56781234,56718234,46781235,46718235 .
$$

The equivalence of the first four properties was given by Deodhar 34. Showing these properties have a pattern avoidance characterization is due to Billey-Warrington [12. Deodhar's theorem extends to all Weyl groups and in each case there is again a pattern avoidance characterization due to Billey-Jones [14].

We should explain Deodhar's terminology $\operatorname{defect}(\sigma)$ and $E(v, w)$ because we believe that they might have important implications for answering Question 2, First, fix a reduced expression for $w$. This corresponds with a string diagram $S$ for $w$. Think of each crossing in the string diagram as optional. Then $E(v, w)$ is the set of all string diagrams $\sigma$ for $v$ obtained from $S$ by choosing some subset of the crossings. The defect of $\sigma$ is the number of times two strings come together that have previously crossed an odd number of times in the string diagram, as one progresses vertically. Thus,

$$
P_{v, w}(t)=\sum_{\sigma \in E(v, w)} t^{\operatorname{defect}(\sigma)}
$$

is precisely the sort of combinatorial formula for the Kazhdan-Lusztig polynomials we would like to have. Deodhar has shown that for every pair $v, w \in S_{n}$ there exists a set of string diagrams $E(v, w)$ for which the same formula holds. The only drawback is that in order to find $E(v, w)$ one must basically compute $P_{v, w}$ using another method first.

The next pattern property due to Tenner concerns a subset of the 321-hexagon avoiding permutations.

Pattern Avoidance Property 7. (Tenner 91) The principal order ideal below w in Bruhat order is isomorphic to a Boolean lattice if and only if $w$ is 321 and 3412 avoiding. Equivalently, the Bott-Samelson resolution of $X_{w}$ is isomorphic to $X_{w}$.

Thus, a permutation is called Boolean if it is 321 and 3412 avoiding. These permutations give rise to a familiar enumerative sequence. 
H. ABE AND S. BILLEY

Theorem 4.20. (Fan [42], West [96]) The number of Boolean permutations in $S_{n}$ is the Fibonacci number $F_{2 n-1}$, e.g. $F_{1}=1, F_{3}=2, F_{5}=5$.

The next property relates Kazhdan-Lusztig polynomials to a filtration on permutations. It was conjectured by Billey-Braden [13] and proved by Woo [99].

Pattern Avoidance Property 8. (Woo-Billey-Weed 99]) The Kazhdan-Lusztig polynomial $P_{i d, w}(1)=2$ if and only if $w$ avoids 653421, 632541, 463152, 526413, 546213, and 465132 and the singular locus of $X_{w}$ has exactly 1 component.

To define a filtration on permutations in a similar way, let's make the following definition.

Definition 4.21. Let $K L_{m}=\left\{w \in S_{\infty} \mid P_{i d, w}(1) \leq m\right\}$.

For example, we know from Property 1 that $K L_{1}$ is the set of permutations avoiding 3412 and 4231. Similarly Billey-Weed used Woo's theorem to show that $K L_{2}$ is characterized by the 66 permutation patterns of length $\leq 8$ below. This result is in an appendix to 99 .

$$
\begin{aligned}
& (45123)(34512)(53412)(52341)(45231) \\
& (3516244)(523614)(526314)(624153)(524613) \\
& (4625513)(526413)(546213)(361452)(461352) \\
& (364152)(463152)(536142)(465132)(426351) \\
& (6325541)(635241)(642531)(653421) \\
& (3612745)(6231745)(6241735)(3416725) \\
& (42366715)(4263715)(4267315)(3712564) \\
& (72331564)(3715264)(3751264)(7523164) \\
& (62551734)(7261453)(3417562)(3517462) \\
& (45177362)(4237561)(5347261)(4275631) \\
& (341227856)(423317856)(34172856) \\
& (422371856)(42731856)(35127846) \\
& (523317846)(52417836)(34128675) \\
& (42318675)(34182675)(42381675) \\
& (42831675)(34186275)(42386175) \\
& (42863175)(35128674)(52318674) \\
& (36128574)(62318574)(52418673) \\
& (62518473)
\end{aligned}
$$

A local ring $R$ is a local complete intersection ( $L C I)$ if it is the quotient of some regular local ring by an ideal generated by a regular sequence. A variety is $L C I$ if every local ring is LCI.

Pattern Avoidance Property 9. (Úlfarsson-Woo 95]) A Schubert variety $X_{w}$ is LCI if and only if $w$ avoids 53241, 52341, 52431, 35142, 42513, and 426153.

Since regular local rings are LCI, smooth varieties are automatically LCI. Furthermore, LCI varieties are Gorenstein and hence Cohen-Macaulay. Thus, being LCI can be viewed as saying that the singularities are in some sense mild. Compare the above criterion with Property 1 (for smoothness) and Property 4 (for Gorenstein property). 
A permutation is vexillary if it avoids 2143, introduced by Lascoux-Schützenberger in 1982 67. The word vexillary is related to flags, hence the choice. We say $w$ is covexillary if $w$ avoids 3412 . There are so many interesting things to say related to vexillary and covexillary permutations so the tenth property has 3 parts.

Pattern Avoidance Property 10-1.

(1) (Edelman-Greene [38]) The number of reduced words for a vexillary permutation $v$ is equal to the number of standard tableaux of shape determined by sorting the lengths of the rows of the diagram of $v$.

(2) (Edelman-Greene [38]) The Stanley symmetric function $F_{v}$ is a Schur function if and only if $v$ is vexillary. Here

$$
F_{v}=\sum_{\mathbf{a}=a_{1} a_{2} \ldots a_{k} \in R(v)} \sum_{i_{1} \leq \cdots \leq i_{k} \in C(\mathbf{a})} x_{i_{1}} x_{i_{2}} \cdots x_{i_{k}}
$$

where $R(v)$ are the reduced words for $v$ and $C(\mathbf{a})$ are the weakly increasing sequences of positive integers such that $i_{j}<i_{j+1}$ if $a_{j}<a_{j+1}$.

(3) (Tenner [91]) The permutation $v$ is vexillary if and only if for every permutation $w$ containing $v$, there exists a reduced decomposition $\mathbf{a} \in R(w)$ containing a shift of some $\mathbf{b} \in R(v)$ as a factor.

The next is a list of properties of vexillary permutations related to geometry of Schubert varieties.

\section{Pattern Avoidance Property 10-2.}

(1) (Fulton [43]) Recall, Fulton's essential set for $w$ is the collection of cells in the diagram of $w$ with no neighbor directly east or south. If $w$ is vexillary, these cells lie on an increasing piecewise linear curve.

(2) (Lascoux [66]) There exists a combinatorial approach to computing the KazhdanLusztig polynomials $P_{v, w}$ when $w$ is covexillary.

(3) (Li-Yong [69]) There exists a combinatorial rule for computing multiplicities for $X_{w}$ when $w$ is covexillary.

We say a permutation $w$ is $k$-vexillary if its Stanley symmetric function $F_{w}$ has at most $k$ terms of Schur functions in its expansion. For example, $F_{2143}=s_{(2)}+s_{(1,1)}$, so 2143 is 2-vexillary.

Pattern Avoidance Property 10-3. (Billey-Pawlowski 9]) The k-vexillary permutations are characterized by a finite set of patterns for all $k$.

For example, if $w$ is a permutation, then the following hold.

(1) $w$ is 2-vexillary if and only if $w$ avoids 35 patterns in $S_{5}, S_{6}, S_{7}, S_{8}$.

(2) $w$ is 3-vexillary if and only if $w$ avoids 91 patterns in $S_{5}, S_{6}, S_{7}, S_{8}$.

The list of 2-vexillary patterns is given as follows:

(32154)(21543)(214365)(241365)(314265)(312645) (214635)(241635) (231564)(215364)(315264)(426153)(5271436)(5173264)(4265173) (2 $547163)(5472163)(5276143)(61832547)(26481537)(64821537)(2658$ $1437)(65821437)(51736284)(51763284)(61837254)(61873254)(2547$ $6183)(54726183)(54762183)(26487153)(64872153)(26587143)(6582$ $7143)(65872143)$.

We have given $10+$ properties of Schubert varieties which are amenable to pattern avoidance in their characterization. This is just the beginning of all the consequences for the Lakshmibai-Sandhya Theorem. In the next section, we will discuss how pattern avoidance extends to other Lie types and Coxeter groups. 
There are two further directions/consequences concerning special families of varieties we should note. First is the $G L_{p} \times G L_{q}$-orbits in the flag variety for $G L_{p+q}$ with rationally smooth closure. These varieties are special cases of the symmetric varieties studied by Springer. McGovern has characterized which symmetric varieties in this case are rationally smooth by using patterns involving a multiset of numbers and + and - signs. See 73 for further details. Similar results are given in type $C$ by McGovern and Trapa [4].

Second, pattern avoidance also comes up in the study of Peterson varieties. The Peterson variety for $\mathbb{C}^{n}$ is the collection of complete flags $F_{\bullet}$ such that $N \cdot F_{i} \subset F_{i+1}$ for all $1 \leq i<n$ where $N$ is a fixed regular nilpotent matrix. Up to isomorphism, the variety is independent of the choice of $N$. Insko and Yong gave a combinatorial description of the singular locus of the Peterson variety which involves the patterns 123 and 2143 among other conditions [55].

\section{Pattern avoidance for Coxeter groups}

In this section, we study pattern avoidance properties for Coxeter groups. First, we recall the definition of Coxeter groups and their basic properties. For details, see [17, 54].

5.1. A quick review on Coxeter groups. A Coxeter graph is a simple graph with vertices $\{1,2, \ldots, n\}$ and edges labeled by $\mathbb{Z}_{\geq 3} \cup \infty$. The Coxeter group associated to a Coxeter graph $G$ is the group generated by $S=\left\{s_{1}, s_{2}, \ldots, s_{n}\right\}$ with relations

(1) $s_{i}^{2}=1$.

(2) $s_{i} s_{j}=s_{j} s_{i}$ if $i, j$ not adjacent in $G$.

(3) $\underbrace{s_{i} s_{j} s_{i} \cdots}_{m(i, j) \text { generators }}=\underbrace{s_{j} s_{i} s_{j} \cdots}_{m(i, j) \text { generators }}$ if $i, j$ connected by edge labeled $m(i, j)<\infty$.

Since a Coxeter group is completely determined by its Coxeter graph, we simply need to draw the graph to refer to the associated Coxeter group. Conventionally, we drop the label 3 from any edge in pictures for simplicity.

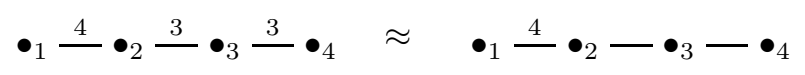

Example 5.1. The following are examples of Coxeter groups.

(1) Dihedral groups: $\operatorname{Dih}_{10}$ is

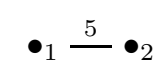

(2) Symmetric groups: $S_{5}$ is

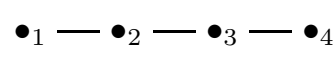

(3) Hyperoctahedral groups: $B_{4}$ is

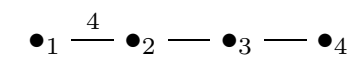

(4) The exceptional Weyl groups: $E_{8}$ is

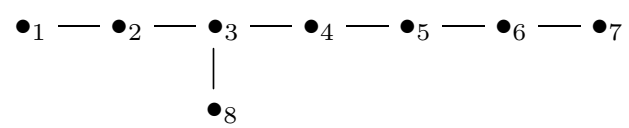

Curiously, the exceptional Weyl group $E_{8}$ appears in string theory and in chemistry related to the symmetry group of the $C_{60}$ molecule and buckyballs [31.

Fix a Coxeter group $W$ with Coxeter graph $G$. The set of reflections $R \subset W$ is the set of all conjugates of the generators,

$$
R=\bigcup_{w \in W} w S w^{-1}
$$

A reduced expression of an element $w \in W$ is an expression $w=s_{i_{1}} \cdots s_{i_{k}}$ as a product of generators in which $k$ is the minimum among such expressions. The length of $w \in W$ is the 
length of a reduced expression for $w$, denoted $\ell(w)$ again. Bruhat order on the Coxeter group $W$ is the transitive closure of the following relation

$$
x \leq y \quad \text { if } \quad \ell(x)<\ell(y) \text { and } x y^{-1} \in R .
$$

It was observed by Chevalley that $x \leq y$ if and only if for any reduced expression $y=$ $s_{i_{1}} s_{i_{2}} \ldots s_{i_{p}}$ there exists a subexpression which is a reduced expression for $x$, in symbols $x=s_{i_{1}}^{\sigma_{1}} s_{i_{2}}^{\sigma_{2}} \ldots s_{i_{p}}^{\sigma_{p}}$ for some mask $\sigma_{1} \ldots \sigma_{p} \in\{0,1\}^{p}$ [30].

There are many expressions for any $w \in W$ as a product of generators, but it is a well known hard problem to tell when two expressions are equal in a group using only generators and relations. Luckily, there is an algorithm of finding a canonical representative for each element of $W$, called the Mozes numbers game. See Mozes 1990, Eriksson-Eriksson 1998, Björner-Brenti [17, 41, 77. Let us briefly explain this game/algorithm here.

Replace each edge $(i, j)$ of $G$ by two opposing directed edges labeled $f_{i j}>0$ (for the edge $i \rightarrow j$ ) and $f_{j i}>0$ (for the edge $j \rightarrow i$ ) so that $f_{i j} f_{j i}=4 \cos ^{2}\left(\frac{\pi}{m(i, j)}\right)$ or $f_{i j} f_{j i}=4$ if $m(i, j)=\infty$. These labels are fixed in the game once chosen. The following is a useful choice since the labels are all integers.

\begin{tabular}{c|c|c}
$m(i, j)$ & $f_{i j}$ & $f_{j i}$ \\
\hline 3 & 1 & 1 \\
4 & 2 & 1 \\
6 & 3 & 1 \\
$\infty$ & 4 & 1
\end{tabular}

Assume that we are given an element $w=s_{i_{1}} s_{i_{2}} \ldots s_{i_{p}} \in W$. The canonical presentation of $w$ is obtained as follows. We first assign value 1 to each vertex $G$. Next, fire the vertex $s_{i_{1}}$. Here, firing the vertex $s_{i}$ is an operation done by adding to the value of each neighbor vertex $j$, the current value at the vertex $i$ multiplied by $f_{i j}$, and then negating the sign of the value of the vertex $i$. We continue to fire the vertices $s_{i_{2}}, s_{i_{3}}, \ldots, s_{i_{p}}$ consecutively. The resulting assignment of values for vertices of $G$, denoted by $G(w)$, provides a canonical presentation of the given $w$. In fact, this algorithm satisfies the following properties:

(1) $G(w)$ only depends on the product $s_{i_{1}} s_{i_{2}} \ldots s_{i_{p}}$ and not on the particular choice of expression.

(2) The vertex $i$ is negative in $G(w)$ if and only $\mathrm{f} w s_{i}<w$.

(3) The vertex $i$ never has value 0 .

Note, the map $G$ is injective but not surjective on the set of all integer assignments to the nodes of the Coxeter graph.

Remark 5.2. For $I \subset S$, it is possible to modify the game to get representatives for $W / W_{I}$ by starting with initial value 0 on vertices in $I$ and 1's elsewhere. Then $w s_{i}=w$ if and only if the vertex $i$ has value 0 in $W / W_{I}$. This is useful for Schubert geometry of Grassmannians and affine Grassmannians.

For a Coxeter group $W$, we can associate to it its root system $\Phi \subset V=\mathbb{R}^{|S|}$ where $\left\{\alpha_{s}: s \in S\right\}$ forms a basis of $V$ [54, Section 5.4]. $W$ acts linearly on $V$ and $\Phi$ is $W$-invariant. We denote by $\Phi_{+}$and $\Phi_{-}$the set of positive roots and the set of negative roots, respectively:

$$
\begin{aligned}
& \Phi_{+}=\left\{\alpha \in \Phi: \alpha=\sum c_{s} \alpha_{s}, c_{s} \geq 0, \forall s \in S\right\}, \\
& \Phi_{-}=\left\{\alpha \in \Phi: \alpha=\sum c_{s} \alpha_{s}, c_{s} \leq 0 \forall s \in S\right\} .
\end{aligned}
$$

It follows that $\Phi=\Phi_{+} \cup \Phi_{-}$(disjoint union). There is a natural bijection between $R$ and $\Phi_{+}$ which we will denote by $r \rightarrow \alpha_{r}$. Then, for $r \in R, w \in W$, we have

$$
w r>w \quad \text { if and only if } w \alpha_{r} \in \Phi_{+} .
$$


Example 5.3. Let $e_{1}, \ldots, e_{n}$ be the standard orthonormal basis of $\mathbb{R}^{n}$. Then the root system of the Weyl groups of classical types are determined by the following description of $\Phi_{+}$.

$$
\begin{array}{ll}
A_{n-1}: & \Phi_{+}=\left\{e_{i}-e_{j}: 1 \leq i<j \leq n\right\} \\
B_{n}: & \Phi_{+}=\left\{e_{i}-e_{j}: 1 \leq i<j \leq n\right\} \cup\left\{e_{i}+e_{j}: 1 \leq i<j \leq n\right\} \cup\left\{e_{i}: 1 \leq i \leq n\right\} \\
C_{n}: & \Phi_{+}=\left\{e_{i}-e_{j}: 1 \leq i<j \leq n\right\} \cup\left\{e_{i}+e_{j}: 1 \leq i<j \leq n\right\} \cup\left\{2 e_{i}: 1 \leq i \leq n\right\} \\
D_{n}: & \Phi_{+}=\left\{e_{i}-e_{j}: 1 \leq i<j \leq n\right\} \cup\left\{e_{i}+e_{j}: 1 \leq i<j \leq n\right\}
\end{array}
$$

The inversion set of $w \in W$ is defined to be $w \Phi_{+} \cap \Phi_{-}$. In type $A_{n-1}$, these roots are in bijection with the inversion set of $w$ defined originally. For a linear function $H: V \longrightarrow \mathbb{R}$, we let

$$
\Pi_{H}=\{\alpha \in \Phi: H(\alpha)>0\} .
$$

This is an intersection of the set of roots with a half space. We say $H$ is generic if $H(\alpha) \neq 0$ for all $\alpha \in \Phi$.

Example 5.4. If $H_{1}: V \longrightarrow \mathbb{R}$ is defined by $H_{1}\left(\alpha_{s}\right)=1$ for all $s \in S$, then $\Pi_{H_{1}}=\Phi_{+}$.

Definition 5.5. For each $w \in W$, set $H_{w}=H_{1} \circ w^{-1}$. Then, we have $\Pi_{H_{w}}=w \Phi_{+}$.

A key fact is that, if $H$ is generic, then $\Pi_{H}=w \Phi_{+}$for some unique $w \in W$. That is, every generic half space determines a unique $w \in W$ whose inversion set is exactly the negative roots in the given half space. Below are the positive roots for two types of Coxeter groups drawn projectively in 2 dimensions. We denote by $\beta_{i j}=e_{i}-e_{j}$ for $A_{3}$.

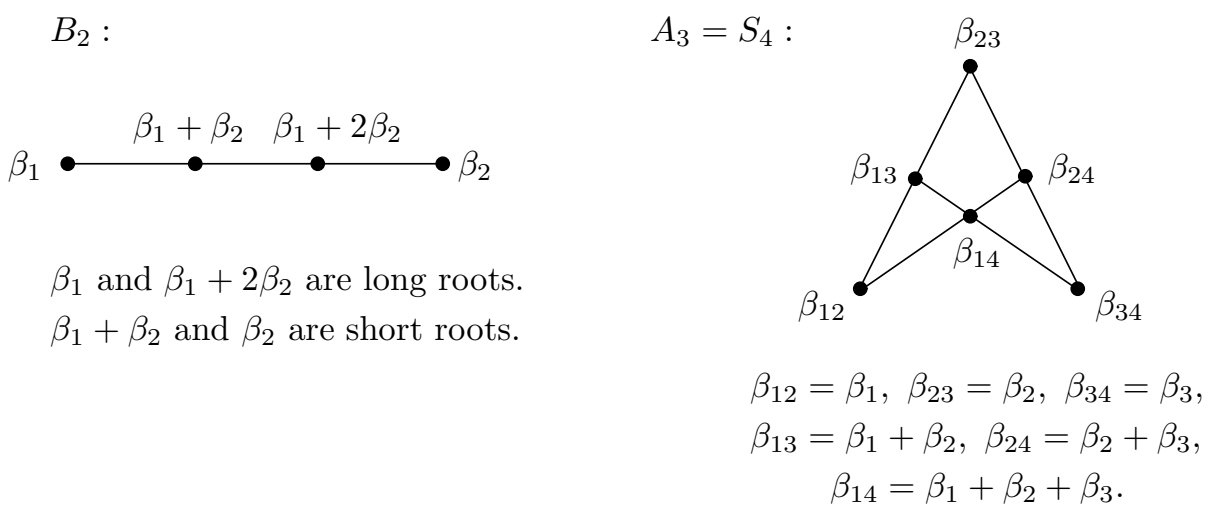

For example, if a given half space $\Pi_{H}$ contains all the positive roots $\beta_{i j}$ 's except for $\beta_{23}$ and $\beta_{24}$, then $\Pi_{H}=\Pi_{H_{w}}$ for $w=2431$.

5.2. Coxeter patterns. Each subset $I \subset S$ generates a subgroup $W_{I}$. A subgroup $W^{\prime} \subset W$ which is conjugate to $W_{I}$ for some $I$ is called a parabolic subgroup. The $W_{I}$ 's themselves are known as standard parabolic subgroups.

A parabolic subgroup $W^{\prime}=x W_{I} x^{-1}$ of $W$ is again a Coxeter group, with simple reflections $S^{\prime}=x I x^{-1}$ and reflections $R^{\prime}=R \cap W^{\prime}$. Note that $S^{\prime} \not \subset S$ unless $W^{\prime}$ is standard.

We denote the length function and the Bruhat-Chevalley order for $\left(W^{\prime}, S^{\prime}\right)$ by $l^{\prime}$ and $\leq^{\prime}$, respectively. If $W^{\prime}=W_{I}$ then

$$
l^{\prime}=\left.l\right|_{W^{\prime}} \text { and } \leq^{\prime}=\leq\left.\right|_{W^{\prime} \times W^{\prime}},
$$

but in general we only have $l^{\prime}(w) \leq l(w)$ and $x \leq^{\prime} y \Longrightarrow x \leq y$. For instance, if $W^{\prime} \subset S_{4}$ is generated by the reflections $r_{23}=1324$ and $r_{14}=4231$, then $r_{23} \leq r_{14}$ although they are not comparable for $\leq^{\prime}$. 
The following theorem/definition generalizes the flattening function for permutations to all Coxeter groups. The following theorem is closely related to a theorem due to Dyer on reflection subgroups [36, Thm. 1.4].

Theorem 5.6. 13] Let $W^{\prime} \subset W$ be a parabolic subgroup. There is a unique function $\mathrm{fl}: W \rightarrow$ $W^{\prime}$, the pattern map for $W^{\prime}$, satisfying the following two properties.

(a) The map $\mathrm{fl}$ is $W^{\prime}$-equivariant: $\mathrm{fl}(w x)=w \mathrm{fl}(x)$ for all $w \in W^{\prime}, x \in W$.

(b) If $\mathrm{fl}(x) \leq^{\prime} \mathrm{fl}(w x)$ for some $w \in W^{\prime}$, then $x \leq w x$.

In particular, fl restricts to the identity map on $W^{\prime}$.

If $W^{\prime}=W_{I}$ is a standard parabolic, then (b) can be strengthened to "if and only if". In this case the result is well-known.

To show uniqueness, note that (a) implies that $\mathrm{fl}$ is determined by the set $\mathrm{fl}^{-1}(1)$, and (b) implies that $\mathrm{fl}^{-1}(1) \cap W^{\prime} x$ is the unique minimal element in $W^{\prime} x$. Existence is more subtle; it is not immediately obvious that the function so defined satisfies (b). We give a construction of a function fl that satisfies (a) and (b).

Recall $V$ is the real vector space spanned by the roots in the root system $\Phi$ associated to the Coxeter group $W$. If $U \subset V$ is a linear subspace, then we use the following notations:

$$
\begin{aligned}
& \Phi^{U}:=\Phi \cap U, \text { a root subsystem of } \Phi, \\
& W^{U} \text { is the group generated by reflections } r_{\alpha} \text { for } \alpha \in \Phi^{U}, \\
& R^{U}:=R \cap W^{U} .
\end{aligned}
$$

One can show that $W^{U}$ is a parabolic subgroup of $W$ assuming $W$ is finite, see [54, §1.12]. Note that not all subgroups of $W$ generated by reflections are parabolic subgroups. For example, for $B_{3}$, the group generated by reflections over the $e_{i}$ 's is not parabolic.

By the uniqueness statement in Theorem [5.6, we can use the sets $\Pi_{H}$ defined earlier to realize $\mathrm{fl}: W \longrightarrow W^{U}$. In fact, $\mathrm{fl}(w)$ is the unique element $x \in W^{U}$ such that

$$
\begin{aligned}
w \Phi_{+} \cap U & =\left\{\alpha \in U \cap \Phi: H_{w}(\alpha)>0\right\} \\
& =\left\{\alpha \in \Phi^{U}: H^{\prime}(\alpha)>0\right\} \text { where } H^{\prime}=\left.H_{w}\right|_{U} \\
& =x \Phi_{+}^{U} .
\end{aligned}
$$

This realization of the flattening map for Weyl groups was first given by Billey-Postnikov [10] even though it was published later than [13. The delay is explained below.

Example 5.7. Let $U=\operatorname{span}\left\langle\beta_{23}, \beta_{34}\right\rangle$. Then $\mathrm{fl}_{U}(2431)=243$. See the picture in the previous example.

\subsection{Applications of Coxeter Patterns. Let us denote}

$G$ : a semisimple simply-connected complex Lie group,

$B \subset G$ : a Borel subgroup,

$T \subset B$ : a maximal torus,

$W=N(T) / T$ : the Weyl group (a finite Coxeter group),

$\Phi \subset V$ : the associated root system

where $N(T)$ is the normalizer of $T$ in $G$. The finite Weyl groups (or root systems) that arise this way have been completely classified into types $A_{n}, B_{n}, C_{n}, D_{n}, E_{6}, E_{7}, E_{8}, F_{4}, G_{2}$. The Bruhat decomposition enables us to partition $G$ using the Borel subgroup and the Weyl group:

$$
G=\bigcup_{w \in W} B w B .
$$

The quotient $G / B$ is called the (generalized) flag manifold, and Schubert cells and Schubert varieties of $G / B$ are

$$
C_{w}=B \cdot w, \quad X_{w}=\overline{B \cdot w}
$$

for each $w \in W$, respectively. 
The next theorem characterizes all smooth Schubert varieties for any semisimple simplyconnected complex Lie group $G$. To state the theorem, we need a few more definitions.

Definition 5.8. A Coxeter group $W$ is stellar if its Coxeter graph has one central vertex and all other vertices are only adjacent to it.

The stellar Coxeter groups corresponding to the Weyl groups of types $A, B, C, D, E, F$ and $G$ (except for $A_{2}$ ) are drawn below where a double edge and a triple edges mean that the label of the corresponding edge is 4 and 6 , respectively. Note, that the Weyl groups of types $B_{n}$ and $C_{n}$ are isomorphic, but the pattern map works slightly differently on each so we list their Dynkin diagram instead of their Coxeter graph.

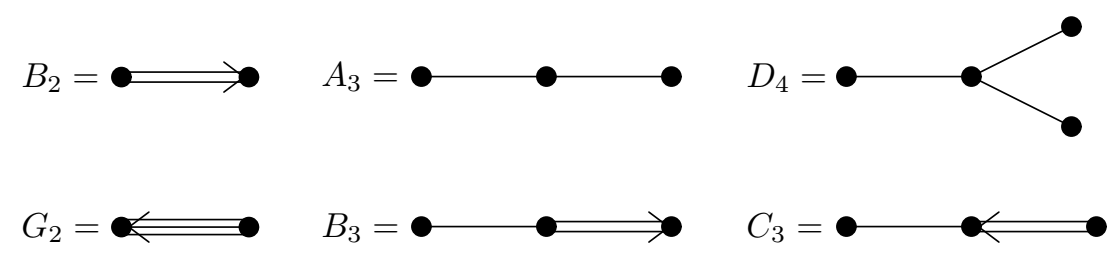

Dynkin diagrams of stellar root systems

Theorem 5.9. (Billey-Postnikov 10]) A Schubert variety $X_{w}$ is smooth if and only if for every stellar parabolic subgroup $W^{U}$, the Schubert variety $X_{v}$ for $v=\mathrm{fl}_{U}(w)$ is smooth in $G^{U} / B^{U}$.

Here $G^{U}$ is a semisimple Lie group with Weyl group $W^{U}$ and $B^{U}$ is one of its Borel subgroups. We remark that $W^{U}$ might not be the same type as $W$. For example, for Weyl groups of type $C$ and $D$ there will appear $W^{U}$ of type $A$. In fact, the type $A$ singular patterns are most common. If the Coxeter graph of $W$ has only edges labeled by 3 's, we say $W$ is simply laced. If $W$ is simply laced, then all of its parabolic subgroups are also simply laced.

It turns out that there are very few patterns for which the corresponding Schubert varieties are singular among stellar reduced, irreducible Weyl groups; 2 patterns in $A_{3}, 1$ pattern in $B_{2}, 6$ patterns in $B_{3}$ and $C_{3}, 1$ pattern in $D_{4}, 5$ patterns in $G_{2}$. Note that all Schubert varieties of type $A_{2}$ are smooth.

Example 5.10. In type $B_{n}$ using the classical pattern avoidance on signed permutations, the smooth Schubert varieties are classified by avoiding the following 17 patterns

$$
\begin{aligned}
& (-2,-1), \\
& (1,2,-3),(1,-2,-3),(-1,2,-3),(2,-1,-3),(-2,1,-3),(3,-2,1) \\
& (2,-4,3,1),(-2,-4,3,1),(3,4,1,2),(3,4,-1,2),(-3,4,1,2) \\
& (4,1,3,-2),(4,-1,3,-2),(4,2,3,1),(4,2,3,-1),(-4,2,3,1) .
\end{aligned}
$$

All length 4 patterns come from $A_{3}$ root subsystems.

Example 5.11. In type $D_{4}$, there are 49 singular Schubert varieties, and the only element which does not comes from $A_{3}$ root subsystems is $w=s_{2} \cdot s_{1} s_{3} s_{4} \cdot s_{2}=\overline{1} 4 \overline{3} 2$. Thus, for all simply laced types, there are only 3 bad patterns to consider: 3412,4231 , and $\overline{1} 4 \overline{3} 2$. It is instructive to look at the singular locus of the Schubert varieties for each of these 3 patterns:

$$
\begin{array}{ll}
\text { Sing } X_{s_{2} s_{1} s_{3} s_{2}}=X_{s_{2}} & \text { (3412 case), } \\
\text { Sing } X_{s_{3} s_{1} s_{2} s_{1} s_{3}}=X_{s_{1} s_{3}} & \text { (4231 case), } \\
\text { Sing } X_{s_{2} s_{1} s_{3} s_{4} s_{2}}=X_{s_{2}} & (\overline{1} 4 \overline{3} 2 \text { case }) .
\end{array}
$$


As we mentioned in Section 4, the definition of a Kazhdan-Lusztig polynomial $P_{v, w}(t)$ easily generalizes to all Coxeter groups. We use these polynomials to define the notion of a rationally smooth Schubert variety. This avoids the more general definition in terms of étale cohomology.

Definition 5.12. A point $v \in X_{w}$ is rationally smooth if and only if $P_{v, w}(t)=1$. A Schubert variety $X_{w}$ is rationally smooth if every point of $X_{w}$ is rationally smooth.

The following theorem as stated is due to Carrell and Peterson. Related results also appear in Jantzen's book [57, Ch.5] in slightly different language.

Theorem 5.13. [27] The following are equivalent.

(1) $X_{w}$ is rationally smooth at $v$.

(2) $P_{v, w}(t)=1$

(3) The Bruhat graph on $[v, w]$ is regular of degree $l(w)-l(v)$.

In the next theorem, the third condition is due to Carrell-Peterson [27]. The fourth condition combines work of Garsharov 44 in type $A$, 6] for types $B$ and $C$, then it was conjectured to hold for all Weyl groups by McGovern and proved by Akyildiz-Carrell [1] for types $D$ and $E$. It can be checked by computer for $F_{4}$ and $G_{2}$ can be done easily. The next condition is due to Oh-Yoo 79. The last condition is due to Slofstra 87.

Theorem 5.14. The following are equivalent for all Weyl groups.

(1) $X_{w}$ is rationally smooth.

(2) $P_{i d, w}(t)=1$

(3) $P_{w}(t)=\sum_{v \leq w} t^{\ell(v)}$ is palindromic.

(4) $P_{w}(t)=\prod\left(1+t+t^{2}+\cdots+t^{e_{i}}\right)$

(5) The Poincaré polynomial $P_{w}(t)$ is equal to the generating function $R_{w}(t)$ for the number of regions $r$ in the complement of the inversion hyperplane arrangement for $w$ weighted by the distance of each region to the fundamental region.

(6) The inversion arrangement for $w$ is free and the number of chambers of the arrangement is equal to the size of the Bruhat interval $[i d, w]$.

For all finite Weyl groups, rational smoothness can be characterized by pattern avoidance.

Theorem 5.15. (Billey-Postnikov [10] $X_{w}$ is rationally smooth if and only for every stellar parabolic subgroup $W^{U}, X_{v}$ for $v=\mathrm{fl}_{U}(w)$ is rationally smooth in $G^{U} / B^{U}$.

Note that there are only 2 patterns in $A_{3}, 6$ patterns of type $B_{3}$ and $C_{3}, 1$ pattern of type $D_{4}$ which should be avoided by $w$ in order for $X_{w}$ to be rationally smooth. The Coxeter pattern map made a very large reduction in the number of patterns one needs to remember for both smoothness and rational smoothness.

Remark 5.16. Smoothness implies rational smoothness. In terms of the patterns characterization, the difference between smoothness and rational smoothness for all Weyl group types is just 6 additional patterns, 1 pattern in $B_{2}$ and 5 patterns of type $G_{2}$.

Outline of proof of Theorems 5.9 and 5.15

- Step 1: For classical types $B, C, D$, use Lakshmibai's characterization of the tangent space basis to get the general smoothness results.

- Step 2: Use an analog of Gasharov's theorem to the factor of Poincaré polynomial for any signed permutation not containing a singular pattern to get the rational smoothness of $B, C, D$ which extends to all finite types. 
- Step 3: Use Kumar's criterion for (rational) smoothness in the nil-Hecke ring to test $G_{2}$ and $F_{4}$ by computer.

- Step 4: Run a massive parallel computation on the 696,729,600 elements $w \in E_{8}$.

- If $w$ has a pattern from type $A$ or $D$, calculate the coefficient of $t^{1}$ and $t^{\ell(w)-1}$ and compare, if different, $w$ is done. If not, calculate the coefficient of $t^{2}$ and $t^{\ell(w)-2}$, etc. Eventually one pair differed in every case.

- If $w$ avoids all patterns from type $A$ or $D$, use analog of Gasharov's algorithm for factoring $P_{w}(t)$.

Note that smoothness of $X_{w}$ automatically implies rational smoothness. Deodhar proved the following property for type $A$, and later Peterson proved that it also holds for type $D$ and $E$ (unpublished). See 28 for a proof. A proof for all finite Weyl group types except $E_{6}, E_{7}, E_{8}$ follows easily from Theorem 5.9 and Theorem 5.15 For $E_{6}, E_{7}, E_{8}$, the Peterson theorem is used in the proof of these two theorems.

Theorem 5.17. (Deodhar, Peterson, Carrel-Kuttler) For types A, D, E, a Schubert variety $X_{w}$ is smooth if and only if it is rationally smooth.

A new proof of Theorem 5.17 has recently been announced by Richmond and Slofstra 85. In fact, they show that every rationally smooth Schubert variety in any finite Lie type is an iterated fibre bundle of Grassmannians. This generalizes the work in type $A$ by Ryan [86, Wolper [98, and Gasharov-Reiner [46] mentioned in Pattern Property 1.

Note that smoothness and rational smoothness are not equivalent for affine type $\widetilde{A}_{n}$ by Mitchell [76] and Billey-Crites [7].

The definition of the Coxeter pattern map also has applications to the geometry of Schubert varieties for Weyl groups and affine Weyl groups. Once again, let $U \subset V$ be a linear subspace. We denote by $M(x, w ; U)$ the set of maximal elements in $[i d, w] \cap W^{U} x$ with respect to a new partial order $\leq_{x}$ defined by

$$
w x \leq_{x} w^{\prime} x \text { if } \mathrm{fl}(w x) \leq^{U} \mathrm{fl}\left(w^{\prime} x\right) .
$$

Theorem 5.18. (Billey-Braden [13]) If $x, w \in W$, then

$$
P_{x, w}(1) \geq \sum_{y \in M(x, w ; U)} P_{y, w}(1) P_{\mathrm{fl}(x), \mathrm{f}(y)}^{U}(1) .
$$

Corollary 5.19. [13] For all $x<w, P_{x, w}(1) \geq P_{\mathrm{fl}(x), \mathrm{fl}(w)}^{U}(1)$.

Historically, Theorem 5.18 and Corollary 5.19 were the first application of simultaneous pattern embedding/flattening on two Coxeter group elements. For $u, v \in S_{m}$ and $x, y \in S_{n}$, if $[u, v]$ interval pattern embeds into $[x, y]$ using indices $1 \leq i_{1}<\ldots<i_{m} \leq n$ then one can construct a subspace $U$ such that $u=\mathrm{fl}_{U}(x), v=\mathrm{fl}_{U}(y)$ by considering all the roots indexed by values in the set $\left\{x_{i_{1}}, \ldots, x_{i_{m}}\right\}$. Thus, Corollary 5.19 implies one direction of the stronger type $A$ result in Theorem 4.10. From this point of view, Theorem 5.18 and Corollary 5.19 were precursors to the notion of interval pattern avoidance introduced in [102].

Woo 100 extended the notion of interval pattern avoidance to other Weyl groups and proved that many of the nice properties in 102 continue to hold. In particular, the analog of Theorem 4.10 holds for all Weyl groups [100, Cor. 3.3]. Furthermore, Woo relates interval pattern embeddings with isomorphism of Richardson varieties which are intersections of two Schubert varieties with respect to two generic flags. 
Theorem 5.20. 100, Thm. 3.1] Let $W^{\prime}, W$ be Weyl groups. Suppose there is some root subsystem embedding which embeds $[u, v] \subset W^{\prime}$ in $[x, w] \subset W$. Then the Richardson varieties $X_{v}^{u}$ and $X_{w}^{x}$ are isomorphic.

Corollary 5.19 also gives rise to filtrations on permutations.

Corollary 5.21. For each $m, K L_{m}=\left\{w \in S_{\infty} \mid P_{i d, w}(1) \leq m\right\}$ is closed under taking patterns.

It is interesting to ask for a geometrical explanation for why (rational) smoothness of Schubert varieties can be characterized by Coxeter patterns. The following theorem proves one direction of this. The other direction is still open: namely, why are patterns from stellar Coxeter graphs enough.

Theorem 5.22. (Billey-Braden [13]) If $X_{\mathrm{fl}(w)}^{U}$ is singular, then $X_{w}$ is singular.

Outline of proof.

- Realize $G^{U} / B^{U}$ as the fixed points of a certain torus action.

- Use a theorem of Fogarty-Norman saying that for all smooth algebraic $T$-schemes $X$ the fixed point scheme $X^{T}$ is smooth.

Several other nice pattern avoidance properties in Coxeter groups are also known:

(1) (Stembridge [89]) The fully commutative elements in types $B$ and $D$ are characterized with signed patterns.

(2) (R.Green [50]) The fully commutative elements in the affine Weyl group of type $A$ are exactly the 321-avoiding elements.

(3) (Reading [82]) Coxeter-sortable elements are characterized and it is shown that they are equinumerous with clusters and with noncrossing partitions.

(4) (Billey-Jones [14]) Deodhar elements for all Weyl groups are characterized.

(5) (Billey-Crites [7]) The rationally smooth Schubert varieties in the affine type A flag manifold are characterized as 3412, 4231 avoiding plus one extra family of twisted spiral varieties. Crites also studied the enumeration of affine permutations indexing rationally smooth Schubert varieties in [33].

(6) (Chen-Crites-Kuttler, manuscript) A Schubert variety $X_{w}$ of affine type A is smooth if and only if $w \in \widetilde{S}_{n}$ avoids 3412 and 4231 . Furthermore, the tangent space to $X_{w}$ at the identity can be described in terms of reflection over real and imaginary roots.

(7) (Matthew Dyer, manuscript) Smooth and rationally smooth Schubert varieties can be detected using rank 2 subvarieties 37.

(8) (Matthew Samuel, manuscript) Affine Schubert varieties for all types can be characterized by patterns using a new version of pattern avoidance for Coxeter groups based on reflection groups.

\section{Computer tools for Schubert Geometry}

In the lecture series that gave rise to this article, we discussed some computer tools for the study of geometry of Schubert varieties and for more general topics in mathematics. The video file of the lecture devoted to the contents of this section is available at the following website.

$$
\text { http://mathsoc.jp/en/videos/2012msj-si.html }
$$

The main ideas presented are pertaining to computer proofs, the Online Encyclopedia of Integer Sequences, the Database of Permutation Pattern Avoidance and Sage. The demos in the lecture are best seen online so we will not include that discussion here. 
We do want to highlight one of the Sage demos discussed, because it is related to some recent developments on marked mesh patterns which unify the descriptions of several pattern avoidance properties for permutations using the language of marked mesh patterns.

Definition 6.1. (Bränden and Claesson [24]) A mesh pattern is a permutation matrix with shaded regions between certain entries.

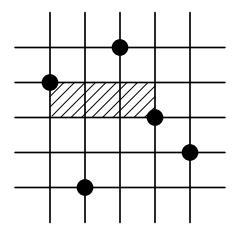

The dots represents 1's in the permutation matrix.

Definition 6.2. (Úlfarsson 94) A marked mesh pattern is a mesh pattern with numbers in the shaded regions.

The next theorem states that we can also use marked mesh patterns for characterizing Schubert varieties. See 94 for details.

Theorem 6.3. (Úlfarsson 94) The smooth, Gorenstein, factorial, defined by inclusions, and 321-hexagon avoiding permutations can be described by marked mesh patterns.

\section{Open Problems}

In addition to the open problems we have mentioned in the text, there are some more open problems concerning pattern avoidance properties. We hope that computer experiments will help the reader to study those problems.

Question 5. (Woo-Yong) Characterize the Gorenstein, LCI and factorial locus of $X_{w}$ using patterns.

Question 6. (From Úlfarsson) Is there a nice generating function to count the number of Gorenstein/LCI permutations or Schubert varieties defined by inclusions, etc.

Question 7. Find a geometric explanation why a finite number of patterns suffice in all cases above.

Question 8. What nice properties does the inversion arrangement have for other pattern avoiding families?

Question 9. $K L_{m}$ is closed under taking patterns by 13 . Can it always be described by a finite set of patterns? Conjectured to be yes by Billey-Weed-Woo.

Question 10. Conjecture (Woo): The Schubert varieties with multiplicity $\leq 2$ can be characterized by pattern avoidance. Can this be extended to a pattern avoidance characterization of Schubert varieties with multiplicity $\leq k$ ? Note, Woo-Yong showed that $\operatorname{mult}_{x}\left(X_{y}\right)=\operatorname{mult}_{u}\left(X_{v}\right)$ if $[u, v]$ interval pattern embeds into [x,y] [102, Cor. 6.15].

Question 11. What other filtrations on the set of all permutations can be characterized by (generalized) patterns?

Question 12. Describe the maximal singular locus of a Schubert variety for other semisimple Lie groups using Coxeter patterns.

Question 13. Give a pattern based algorithm to produce the factorial and/or Gorenstein locus of a Schubert variety in other types. 
Question 14. Is there a nice generating function to count the number of smooth, factorial and/or Gorenstein permutations in other types?

Question 15. What is the right notion of patterns for GKM spaces?

Question 16. Say $X_{w}$ is combinatorially smooth if $\ell(w)=\#\left\{t_{i j}: t_{i j} \leq w\right\}$. In $S_{n}$ combinatorially smooth is equivalent to smooth by the Lakshmibai-Seshadri Theorem. However, for other Weyl groups this is a weaker notion than rational smoothness. Characterize the combinatorially smooth Schubert varieties by generalized pattern avoidance.

Question 17. Can Lakshmibai's characterization of the tangent space basis for $B, C$ and $D$ be translated into signed patterns or a signed variation on marked mesh patterns.

Question 18. What is the analog of marked mesh patterns for other types?

Question 19. What is the Möbius function for the poset of pattern containment on $S_{\infty}$ ? See the excellent survey by Einar Steingrimmson [88, Sect. 5] for more details on this and other pattern related problems.

Question 20. Which of the many pattern avoidance related theorems on Schubert varieties have analogs for other interesting families of varieties such as the $G L_{p} \times G L_{q}$-orbit closures of the flag manifold or the Peterson varieties as mentioned at the end of Section 44. See [23, 52, 55, 73, 74] for further details.

Question 21. More generally, what other types of theorems have canonical representations which might lead to more computer database tools? For example, how about hypergeometric series, integer sequences, patterns? See [11] for more discussion on this topic.

Acknowledgments. We offer our immense gratitude to those who helped in preparation of the lecture series and this article including Michael Albert, Andrew Crites, Jack Lee, Monty McGovern, Julia Pevtsova, Brendan Pawlowski, Ed Richmond, Sudeshna Sen, William Slofstra, Joshua Swanson, Henning Úlfarsson, Alex Woo, Alex Yong, and all of the organizers of the MSJ summer program Megumi Harada, Takeshi Ikeda, Shizuo Kaji, Toshiaki Maeno, Mikiya Masuda, Hiroshi Naruse, Toru Ohmoto, Norio Iwase. We also want to thank the students in Osaka City University who helped organize the conference, the audience who attended the lectures at the conference and the readers of this article. Kokoroyori Kannsha Itashimasu. Finally, we greatly appreciate the corrections and comments of an anonymous referee.

\section{REFERENCES}

[1] Akyildiz, E., And Carrell, J. B. Betti numbers of smooth Schubert varieties and the remarkable formula of Kostant, Macdonald, Shapiro, and Steinberg. Michigan Math. J. 61, 3 (2012), 543-553.

[2] Akyildiz, E., Lascoux, A., And Pragacz, P. Cohomology of Schubert subvarieties of $G L_{n} / P . J$. Differential Geom. 35 (1992), 511-519.

[3] Albert, M. H., Aldred, R. E. L., Atkinson, M. D., and Holton, D. A. Algorithms for pattern involvement in permutations. In in Algorithms and Computation, 12th International Symposium, ISAAC 2001, Proceedings LNCS 2223 (2001), pp. 355-366.

[4] Albert, M. H., and Brignall, R. Enumerating indices of Schubert varieties defined by inclusions. ArXiv e-prints (Jan. 2013).

[5] Beilinson, A., and Bernstein, J. Localization of g-modules. C. R. Acad.Sci. Paris Ser. I Math 292 (1981), 15-18.

[6] Billey, S. Pattern avoidance and rational smoothness of schubert varieties. Adv. in Math. 139, 1 (1998), $141-156$.

[7] Billey, S., And CRites, A. Pattern characterization of rationally smooth affine Schubert varieties of type A. J. of Algebra 361 (2012), 107-133.

[8] Billey, S., And Lakshmibai, V. Singular Loci of Schubert Varieties. No. 182 in Progress in Mathematics. Birkhäuser, 2000. 
[9] Billey, S., and Pawlowski, B. Permutation patterns, Stanley symmetric functions, and generalized Specht modules. to appear J. Combinatorial Theory, Series A (2013).

[10] Billey, S., And Postnikov, A. Smoothness of Schubert varieties via patterns in root subsystems. Adv. in Appl. Math. 34, 3 (2005), 447-466.

[11] Billey, S., And Tenner, B. Fingerprint databases for theorems. Notices. Amer. Math. Soc. 60, 8 (2013), 1034-1039.

[12] Billey, S., And Warrington, G. Kazhdan-Lusztig polynomials for 321-hexagon-avoiding permutations. J. Alg. Comb. 13 (2001), 111-136.

[13] Billey, S. C., And Braden, T. Lower bounds for Kazhdan-Lusztig polynomials from patterns. Transform. Groups 8, 4 (2003), 321-332.

[14] Billey, S. C., And Jones, B. C. Embedded factor patterns for Deodhar elements in Kazhdan-Lusztig theory (submitted). arXiv:math.CO/0612043 (2006).

[15] Billey, S. C., ANd Tenner, B. E. Fingerprint databases for theorems. Notices of the AMS 60, 8 (2013).

[16] Billey, S. C., And Warrington, G. S. Maximal singular loci of Schubert varieties in SL(n)/B. Trans. Amer. Math. Soc. 355, 10 (2003), 3915-3945 (electronic).

[17] Björner, A., And Brenti, F. Combinatorics of Coxeter groups, vol. 231 of Graduate Texts in Mathematics. Springer, New York, 2005.

[18] Bose, P., Buss, J. F., And Lubiw, A. Pattern matching for permutations. Information Processing Letters 65, 5 (1998), $277-283$.

[19] Bousquet-Mélou, M., and Butler, S. Forest-like permutations. Ann. Comb. 11, 3-4 (2007), 335-354.

[20] Braden, T., and MacPherson, R. From moment graphs to intersection cohomology. Mathematische Annalen 321 (2001), 533-551.

[21] Brenti, F. The intersection cohomology of Schubert varieties is a combinatorial invariant. European J. Combin. 25, 8 (2004), 1151-1167.

[22] Brenti, F., Caselli, F., And Marietti, M. Special matchings and Kazhdan-Lusztig polynomials. Adv. Math. 202, 2 (2006), 555-601.

[23] Brion, M. Rational smoothness and fixed points of torus actions. Transform. Groups 4, $2-3$ (1999), $127-156$.

[24] Bränden, P., And Claesson, A. Mesh patterns and the expansion of permutation statistics as sums of permutation patterns. Electron. J. Combin. 18 (2011), 1-14.

[25] Brylinski, J.-L., and Kashiwara, M. Kazhdan-Lusztig conjectures and holonomic systems. Invent. Math. 64 (1981), 387-410.

[26] Carrell, J. B. Some remarks on regular weyl group orbits and the cohomology of schubert varieties. Contemporary Mathematics 139 (1992).

[27] Carrell, J. B. The Bruhat graph of a Coxeter group, a conjecture of Deodhar, and rational smoothness of Schubert varieties. Proceedings of Symposia in Pure Math. 56, Part 1 (1994), 53-61.

[28] Carrell, J. B., And Kuttler, J. Singular points of $t$-varieties in $g / p$ and the Peterson map. Invent. Math. 151 (2003), 353-379.

[29] Chevalley, C. Classification de groupes de lie algébriques, séminaire. Secrétariat mathématique II. (1958), 1956-58. rue Pierre-Curie, Paris.

[30] Chevalley, C. Sur les Décompositions Cellulaires des Espaces G/B. Proceedings of Symposia in Pure Mathematics 56, 1 (1994).

[31] Chung, F., Kostant, B., And Sternberg, S. Groups and the buckyball. In Lie Theory and geometry, vol. 123 of Progr. in Math. Birkhauser, 1994, pp. 97-126.

[32] Cortez, A. Singularités génériques et quasi-résolutions des variétés de Schubert pour le groupe linéaire. Adv. Math. 178, 2 (2003).

[33] CRites, A. Enumerating pattern avoidance for affine permutations. Electron. J. Combin. 17 (2010).

[34] Deodhar, V. V. A combinatorial setting for questions in Kazhdan-Lusztig theory. Geom. Dedicata 36, 1 (1990), 95-119.

[35] DU Cloux, F. Rigidity of Schubert closures and invariance of Kazhdan-Lusztig polynomials. Adv. Math. 180, 1 (2003), 146-175.

[36] Dyer, M. On the "Bruhat graph" of a Coxeter system. Compositio Math. 78, 2 (1991), 185-191.

[37] Dyer, M. Rank two detection of singularities of Schubert varieties. manuscript (2001).

[38] Edelman, P., And Greene, C. Balanced Tableaux. Adv. Math. 63 (1987), 42-99.

[39] Ehresmann, C. Sur la topologie de certains espaces homogènes. Ann. of Math. (2) 35, 2 (1934), 396-443.

[40] Elias, B., and Williamson, G. The Hodge theory of Soergel bimodules. ArXiv e-prints (Dec. 2012).

[41] Eriksson, H., and Eriksson, K. Affine Weyl groups as infinite permutations. Electron. J. Combin. 5 (1998), Research Paper 18, 32 pp. (electronic).

[42] FAn, C. K. Schubert varieties and short braidedness. Transform. Groups 3, 1 (1998), 51-56.

[43] Fulton, W. Flags, Schubert polynomials, degeneracy loci, and determinantal formulas. Duke Math. J. 65 (1992), 381-420. 
[44] Gasharov, V. Factoring the Poincaré polynomials for the Bruhat order on $s_{n}$. Combinatorial Theory, Series A 83 (1998), 159-164.

[45] Gasharov, V. Sufficiency of Lakshmibai-Sandhya singularity conditions for Schubert varieties. Compositio Math. 126, 1 (2001), 47-56.

[46] Gasharov, V., and Reiner, V. Cohomology of smooth Schubert varieties in partial flag manifolds. Journal of the London Mathematical Society (2) 66, 3 (2002), 550-562.

[47] Geck, M., And Kim, S. Bases for the Bruhat-Chevalley order on all finite Coxeter groups. J. Algebra 197 (1997), 278-310.

[48] Goresky, M., Kottwitz, R., and MacPherson, R. Equivariant cohomology, Koszul duality, and the localization theorem. Invent. Math. 131, 1 (1998), 25-83.

[49] Goresky, M., and MacPherson, R. Intersection homology-ii. Invent. Math. 71 (1983), 77-129.

[50] Green, R. On 321-avoiding permutations in affine Weyl groups. J. Alg. Comb. 15, 3 (2002), 241-252.

[51] Guillemot, S., and Marx, D. Finding small patterns in permutations in linear time. ArXiv e-prints (July 2013).

[52] Hultman, A. Criteria for rational smoothness of some symmetric orbit closures. In 22nd International Conference on Formal Power Series and Algebraic Combinatorics (FPSAC 2010), Discrete Math. Theor. Comput. Sci. Proc., AN. Assoc. Discrete Math. Theor. Comput. Sci., Nancy, 2010, pp. 319-330.

[53] Hultman, A., Linusson, S., Shareshian, J., and Sjöstrand, J. From Bruhat intervals to intersection lattices and a conjecture of Postnikov. J. Comb. Theory, Series A 119 (2009), 564-580.

[54] Humphreys, J. E. Reflection groups and Coxeter groups, vol. 29 of Cambridge Studies in Advanced Mathematics. Cambridge University Press, Cambridge, 1990.

[55] Insko, E., And Yong, A. Patch ideals and Peterson varieties. Transform. Groups 17, 4 (2012), 10111036.

[56] Irving, R. S. The socle filtration of a Verma module. Ann. Sci. École Norm. Sup. (4) 21, 1 (1988), $47-65$.

[57] Jantzen, J. C. Moduln mit einem höchsten Gewicht, vol. 750 of Lecture Notes in Mathematics. Springer, Berlin, 1979.

[58] Kassel, C., Lascoux, A., And Reutenauer, C. The singular locus of a Schubert variety. J. Algebra 269, 1 (2003), 74-108.

[59] Kazhdan, D., And Lusztig, G. Representations of Coxeter groups and Hecke algebras. Invent. Math. 53, 2 (1979), 165-184.

[60] Kazhdan, D., And Lusztig, G. Schubert varieties and Poincaré duality. Proc. Symp. Pure. Math., A.M.S. 36 (1980), 185-203.

[61] Klein, A., Lewis, J., And Morales, A. Counting matrices over finite fields with support on skew Young diagrams and complements of Rothe diagrams. J. Alg. Comb. (2013).

[62] Knuth, D. E. The art of computer programming, second ed. Addison-Wesley Publishing Co., Reading, Mass.-London-Amsterdam, 1975. Volume 1: Fundamental algorithms, Addison-Wesley Series in Computer Science and Information Processing.

[63] Kumar, S. The nil Hecke ring and singularity of Schubert varieties. Inventiones Math. 123 (April 1996), 471-506.

[64] Lakshmibai, V., And Sandhya, B. Criterion for smoothness of Schubert varieties in $S L(n) / B$. Proc. Indian Acad. Sci. (Math Sci.) 100, 1 (1990), 45-52.

[65] Lakshmibai, V., And Seshadri, C. S. Singular locus of a Schubert variety. Bull. Amer. Math. Soc. (N.S.) 11, 2 (1984), 363-366.

[66] Lascoux, A. Polynômes de Kazhdan-Lusztig pour les variétés de Schubert vexillaires. C. R. Acad. Sci. Paris Sér. I Math. 321, 6 (1995), 667-670.

[67] Lascoux, A., and Schützenberger, M.-P. Polynômes de Schubert. Comptes Rendus des Séances de l'Académie des Sciences. Série I. Mathématique 294 (1982), 447-450.

[68] Lewis, J., And Morales, A. Combinatorics of diagrams of permutations. In 26th International Conference on Formal Power Series and Algebraic Combinatorics (FPSAC 2014), Discrete Math. Theor. Comput. Sci. Proc., AN. Assoc. Discrete Math. Theor. Comput. Sci., Nancy, 2014.

[69] Li, L., AND Yong, A. Some degenerations of Kazhdan-Lusztig ideals and multiplicities of Schubert varieties. Advances in Mathematics 229 (2012), 633-667.

[70] Macdonald, I. Notes on Schubert Polynomials, vol. 6. Publications du LACIM, Université du Québec à Montréal, 1991.

[71] Madras, N., And Liu, H. Random pattern-avoiding permutations. In Algorithmic probability and combinatorics, vol. 520 of Contemp. Math. Amer. Math. Soc., Providence, RI, 2010, pp. 173-194.

[72] Manivel, L. Le lieu singulier des variétés de Schubert. Internat. Math. Res. Notices, 16 (2001), 849-871.

[73] McGovern, W. M. Closures of K-orbits in the flag variety for U(p,q). ArXiv e-prints (May 2009).

[74] McGovern, W. M., And Trapa, P. E. Pattern avoidance and smoothness of closures for orbits of a symmetric subgroup in the flag variety. J. Algebra 322, 8 (2009), 2713-2730. 
[75] Mclarnan, T. J., and Warrington, G. S. Counterexamples to the 0-1 conjecture. Represent. Theory 7 (2003), 181-195 (electronic).

[76] Mitchell, S. A. A filtration of the loops on $\mathrm{SU}(n)$ by Schubert varieties. Math. Z. 193, 3 (1986), $347-362$.

[77] Mozes, S. Reflection processes on graphs and Weyl groups. J. Combin. Theory Ser. A 53, 1 (1990), $128-142$.

[78] Оh, S., Postnikov, A., And Yoo, H. Bruhat order, smooth Schubert varieties, and hyperplane arrangements. J. Comb. Theory, Series A 115 (2008), 1156-166.

[79] Оh, S., AND Yoo, H. Bruhat order,rationally smooth schubert varieties, and hyperplane arrangements. In 22nd International Conference on Formal Power Series and Algebraic Combinatorics (FPSAC 2010), Discrete Math. Theor. Comput. Sci. Proc., AN. Assoc. Discrete Math. Theor. Comput. Sci., Nancy, 2010, pp. 833-840.

[80] Polo, P. Construction of arbitrary Kazhdan-Lusztig polynomials in symmetric groups. Represent. Theory 3 (1999), 90-104 (electronic).

[81] Pratt, V. R. Computing permutations with double-ended queues, parallel stacks and parallel queues. In Proceedings of the fifth annual ACM symposium on Theory of computing (1973), STOC '73, ACM, pp. 268-277.

[82] Reading, N. Clusters, Coxeter-sortable elements and noncrossing partitions. Trans. Amer. Math. Soc. 359 (2007), 5931-5958.

[83] Reiner, V., Woo, A., And Yong, A. Presenting the cohomology of a Schubert variety. Trans. Amer. Math. Soc. 363 (2011), 521-543.

[84] Richmond, E., And Slofstra, W. Rationally smooth elements of Coxeter groups and triangle group avoidance. ArXiv e-prints (June 2012).

[85] Richmond, E., And Slofstra, W. Billey-Postnikov decompositions and the fibre bundle structure of Schubert varieties. manuscript, March 2014.

[86] Ryan, K. M. On Schubert varieties in the flag manifold of $s l(n, \mathbb{C}$. Math. Ann. (1987).

[87] Slofstra, W. Rationally smooth Schubert varieties and inversion hyperplane arrangements. ArXiv e-prints (Dec. 2013).

[88] Steingrímsson, E. Some open problems on permutation patterns. London Mathematical Society Lecture Note Series, 239-263 (2013).

[89] Stembridge, J. The enumeration of fully commutative elements of Coxeter groups. J. Alg. Comb. 7, 3 (1998), 291-320.

[90] Tarjan, R. Sorting using networks of queues and stacks. J. Assoc. Comput. Mach. 19 (1972), 341-346.

[91] Tenner, B. Pattern avoidance and the Bruhat order. J. Comb. Theory, Series A 114 (2007), 888-905.

[92] Tenner, B. E. Database of permutation pattern avoidance. published electronically at http://math.depaul.edu/bridget/patterns.html Nov 2013.

[93] Tymoczko, J. S. Billey's formula in combinatorics, geometry, and topology. ArXiv e-prints (Sept. 2013).

[94] Úlfarsson, H. A unification of permutation patterns related to Schubert varieties. Pure Mathematics and Applications 22, 2 (2011), 273-296.

[95] Ulfarsson, H., AND Woo., A. Which schubert varieties are local complete intersections? Proc. London Math. Soc. 107, 5 (2013), 1004-1052.

[96] West, J. Generating trees and forbidden subsequences. Discrete Math. 157 (1996), 363-374.

[97] Wikipedia. Kazhdan-Lusztig polynomial — Wikipedia, the free encyclopedia, 2013. [Online; accessed 1-October-2013].

[98] Wolper, J. S. A combinatorial approach to the singularities of Schubert varieties. Adv. Math 76 (1989), 184-193.

[99] Woo, A. Permutations with Kazhdan-Lusztig polynomial $P_{i d, w}(q)=1+q^{h}$. Electron. J. Combin. 16, 2, Special volume in honor of Anders Björner (2009), Research Paper 10, 32. With an appendix by Sara Billey and Jonathan Weed.

[100] Woo, A. Interval pattern avoidance for arbitrary root systems. Canad. Math. Bull. 53, 4 (2010), 757762.

[101] Woo, A., And Yong, A. When is a Schubert variety Gorenstein? Adv. in Math 207, 1 (2006), 205-220.

[102] Woo, A., ANd Yong, A. Governing singularities of Schubert varieties. J. Algebra 320, 2 (2008), 495-520. 
Department of Mathematics, Faculty of Science and Engineering, Waseda University, 3-4-1 OKubo, ShINJuku, TOKYo 169-8555 JAPAN

E-mail address: hirakuabe@globe.ocn.ne.jp

Department of Mathematics, Padelford C-445, University of Washington, Box 354350, Seattle, WA 98195-4350

E-mail address: billey@math.washington.edu 